\title{
CHANGEMENTS DE BASE EXPLICITES DES REPRÉSENTATIONS SUPERCUSPIDALES DE $U(1,1)\left(F_{0}\right)$
}

\author{
LAURE BLASCO
}

\begin{abstract}
RÉSumÉ. Soit $F_{0}$ un corps local non archimédien de caractéristique nulle et de caractéristique résiduelle impaire. On décrit explicitement les changements de base des représentations supercuspidales de $U(1,1)\left(F_{0}\right)$. C'est une étape vers la description du changement de base des paquets endoscopiques supercuspidaux de $U(2,1)\left(F_{0}\right)$.
\end{abstract}

Bien que le titre n'en dise rien, cet article fait suite à [4] dans lequel nous décrivons explicitement le changement de base stable de certaines représentations supercuspidales du groupe unitaire $U(2,1)\left(F_{0}\right)$ relativement à $F$ au groupe linéaire $G L(3, F)$ où $F_{0}$ est un corps $p$-adique de caractéristique résiduelle impaire et $F$ une extension quadratique de $F_{0}$. Cette description repose sur la classification des représentations supercuspidales par la théorie des types de $\mathrm{C}$. Bushnell et Ph. Kutzko et sur les travaux de J. Rogawski [18].

Plus précisément, dans [4], n'est explicité que le changement de base stable de paquets cuspidaux de cardinal 1 (et on pense qu'ils y sont tous). Il reste donc à faire l'analogue pour les paquets cuspidaux endoscopiques, ce qui se réalise en deux temps [18, Ch.4, §2] :

(1) déterminer les images par l'application de transfert des représentations de carré intégrable du groupe $U(1,1)\left(F_{0}\right) \times U(1)\left(F_{0}\right)$ au groupe $U(2,1)\left(F_{0}\right)$;

(2) décrire le changement de base "labile" (ou "instable") des représentations supercuspidales de $U(1,1)\left(F_{0}\right)$ au groupe $G L(2, F)$.

On conclut alors grâce aux résultats de J. Rogawski [18, prop. 13.2.2(c)]. Notons que pour la représentation de Steinberg de $U(1,1)\left(F_{0}\right)$ et ses tordues par un caractère, la deuxième étape est faite dans [18] (prop. 11.4.1 et démonstration de la prop. 12.4.1).

Ce texte présente la réalisation du point (2). Elle repose sur la liste des types de $U(1,1)\left(F_{0}\right)$ obtenue dans l'annexe de [3], sur les résultats concernant $G L(2, F)$ exposés dans les chapitres 5 et 8 de [7] et sur ceux du chapitre 11 de [18]. Comme dans le cas de $U(2,1)\left(F_{0}\right)$, on détermine le changement de base stable dont l'identité de caractères ne dépend que des classes de conjugaison et conjugaison tordue stables, le changement de base labile s'en déduisant aisément puisqu'il est explicitement relié au précédent ([18, §11.4] ou voir \$1). La compatibilité du changement de base à la torsion par un caractère permet de se restreindre aux représentations supercuspidales de niveau minimal parmi leurs tordues par un caractère.

Date: 7 septembre 2009 .

2000 Mathematics Subject Classification. Primary: 22E50, Secondary: 11F70. 
Dans le cas de $U(1,1)\left(F_{0}\right)$, les paquets supercuspidaux sont bien connus : on en donne une description "typique" dans le paragraphe2, Les paquets endoscopiques sont précisément les paquets de cardinal 2 [18, prop. 11.1.1(a)] et sont formés, en niveau strictement positif, de représentations cuspidales scindées (c'est-à-dire celles dont le type provient d'une strate gauche fondamentale scindée suivant un sous-groupe de Levi non rationnel sur $F_{0}$ ). Les représentations supercuspidales de niveau 0 sont toutes dans des paquets de cardinal 2 si $F$ est non ramifiée sur $F_{0}$ et toutes sauf deux dans des paquets singletons si $F$ est ramifiée sur $F_{0}$.

Par contre, il est plus difficile de distinguer les images des deux changements de base, toutes deux formées de représentations admissibles, invariantes sous l'action du groupe de Galois de $F / F_{0}$ et de caractère central trivial sur $F_{0}^{\times}$. Pour un paquet endoscopique, emprunter la "voie" par l'application transfert de $U(1)\left(F_{0}\right) \times U(1)\left(F_{0}\right)$ à $U(1,1)\left(F_{0}\right)$ et utiliser le changement de base de $U(1)\left(F_{0}\right)$ à $F^{\times}$permet d'ignorer cette question. Mais pour un paquet singleton où l'on établit le changement de base en vérifiant une identité de caractères entre représentations de $U(1,1)\left(F_{0}\right)$ et de $G L(2, F)$ (voir (4.6.1)), repérer les représentations supercuspidales de $G L(2, F)$ qui appartiennent à l'image du changement de base stable est un point crucial.

Dans le paragraphe 3, on détermine le changement de base stable des paquets endoscopiques en empruntant la méthode de Y. Flicker [9] puis on conclut grâce à [18, prop. 11.4.1(a)]. Dans le paragraphe 4, on décrit les changements de base des paquets non endoscopiques en suivant la même démarche que celle exposée dans [4] dont on ne reprend pas tous les détails. L'ensemble des résultats est présenté aux corollaire 3.4 et théorème 4.5 .

Le dernier paragraphe expose un calcul technique permettant de comparer les caractères de représentations d'un groupe et de l'un de ses sous-groupes. Il est utilisé à plusieurs reprises dans les paragraphes précédents.

Les méthodes utilisées n'ont pas d'originalité mais fournissent les résultats désirés, non écrits jusque-là, sous une forme que nous pourrons exploiter ultérieurement et sous un minimum d'hypothèses $\left(F_{0}\right.$ de caractéristique nulle et de caractéristique résiduelle impaire). Pour ces raisons, on inclut les représentations cuspidales de niveau 0 déjà étudiées par J. Adler et J. Lansky ([1] et [2]).

Je remercie Corinne Blondel pour ses encouragements patients et sa relecture critique.

\section{Notations}

Soient $F_{0}$ un corps local non archimédien, de caractéristique nulle et de caractéristique résiduelle différente de deux et $F$ une extension quadratique (séparable) de $F_{0}$ d'indice de ramification $e_{0}$ et dont le groupe de Galois est noté $\Gamma: \Gamma=\left\{1,{ }^{-}\right\}$.

On désigne par $\mathfrak{o}_{0}$ (resp. o) l'anneau des entiers de $F_{0}$ (resp. $F$ ), $\mathfrak{p}_{0}$ (resp. $\mathfrak{p})$ l'idéal maximal de $\mathfrak{o}_{0}$ (resp. o) et $\varpi_{0}($ resp. $\varpi)$ une uniformisante de $\mathfrak{p}_{0}$ (resp. p). On choisit les uniformisantes $\varpi$ et $\varpi_{0}$ telles que : $\varpi=\varpi_{0}$ si $e_{0}=1, \varpi$ est de trace nulle et de norme $\varpi_{0}$ si $e_{0}=2$. On note $k_{0}$ et $k$ les corps résiduels de $F_{0}$ et $F$ respectivement et $q$ le cardinal de $k_{0}$. 
Pour une extension $E$ de $F_{0}$, on conserve les mêmes notations que pour $F$, cette fois indexées par $E$. Si $L$ est une sous-extension de $E$, on désigne par $E_{\mid L}^{1}$ le groupe des éléments de $E$ dont la norme dans $L$ est 1.

On fixe un caractère additif $\psi_{0}$ de $F_{0}$, de conducteur $\mathfrak{p}_{0}$. Sa composée avec la trace $\operatorname{tr}_{F / F_{0}}$ est un caractère $\psi$ de $F$ de conducteur $\mathfrak{p}$. On fixe également un prolongement à $F^{\times}$du caractère $\omega_{F / F_{0}}$ de $F_{0}^{\times}$associé à $F / F_{0}$ par la théorie du corps de classes. On choisit ce prolongement, noté $\mu$, égal à $x \mapsto(-1)^{\operatorname{val}_{F}(x)}$ si $F$ n'est pas ramifiée sur $F_{0}$; trivial sur $1+\mathfrak{p}$ et égal à la constante de Langlands $\lambda_{F_{\mid F_{0}}}\left(\psi_{0}\right)$ en $\varpi$ si $F$ est ramifiée sur $F_{0}$ (voir par exemple [7, §34.3].

Si $\chi$ est un caractère de $F_{\mid F_{0}}^{1}$, on désigne par $\tilde{\chi}$ le caractère de $F^{\times}$défini par : $\widetilde{\chi}(x)=\chi\left(\frac{x}{\bar{x}}\right), x \in F^{\times}$. L'application $\chi \mapsto \widetilde{\chi}$ n'est autre que le changement de base stable de $U(1)\left(F_{0}\right)$ à $G L(1, F)$.

Soient $V$ un $F$-espace vectoriel de dimension 2 muni d'une forme hermitienne "isotrope" non dégénérée $<,>, G=U(1,1)\left(F_{0}\right)$ son groupe d'isométries et $\widetilde{G}=G L(2, F)$ son groupe d'automorphismes. On note $Z$ le centre de $U(1,1): Z\left(F_{0}\right)$ s'identifie à $F_{\mid F_{0}}^{1}$ et $Z(F)$ à $F^{\times}$.

Le groupe $\Gamma$ agit sur $\widetilde{G}$ par : l'élément non trivial de $\Gamma$ transforme un élément $g$ de $\widetilde{G}$ en $\tau(g):=\sigma(g)^{-1}$ où $\sigma$ désigne l'involution définie $\operatorname{sur}^{\operatorname{End}_{F} V \text { et }}$ associée à $<,>$. Alors, $G$ n'est autre que le groupe des points de $\widetilde{G}$ fixes sous $\Gamma: G=\widetilde{G}^{\tau}$.

On fixe une base hyperbolique $\mathcal{B}=\left(e_{-1}, e_{1}\right)$ de $V$ et on note $g_{0}$ la similitude de matrice $\left(\begin{array}{cc}1 & 0 \\ 0 & \alpha_{0}\end{array}\right)$ où $\alpha_{0}=\varpi_{0}$ si $F$ n'est pas ramifiée, $\alpha_{0} \in \mathfrak{o}_{0}^{\times}$n'est pas une norme si $F$ est ramifiée. Alors le groupe $G U(1,1)\left(F_{0}\right)$ des similitudes unitaires de $V$ est la réunion de $Z(F) G$ et $g_{0} Z(F) G$.

On fixe également un élément $\varepsilon$ de $F: \varepsilon$ est une unité de trace nulle si $F$ n'est pas ramifiée sur $F_{0}, \varpi$ sinon. On note également $\varepsilon$ l'élément de $\widetilde{G}$ dont la matrice dans $\mathcal{B}$ est $\left(\begin{array}{ll}1 & 0 \\ 0 & \varepsilon\end{array}\right)$. Alors l'application $\Phi$ définie par :

$$
g \mapsto{ }^{\varepsilon} g:=\left(\begin{array}{ll}
1 & 0 \\
0 & \varepsilon
\end{array}\right) g\left(\begin{array}{ll}
1 & 0 \\
0 & \varepsilon
\end{array}\right)^{-1}
$$

est un isomorphisme entre $G Z(F)$ et $G L\left(2, F_{0}\right)^{+} Z(F)$ qui identifie $S U(1,1)\left(F_{0}\right)$ et $S L_{2}\left(F_{0}\right)$. Rappelons que : $G L\left(2, F_{0}\right)^{+}=\left\{g \in G L\left(2, F_{0}\right) \mid\right.$ dét $\left.g \in N_{F \mid F_{0}}\left(F^{\times}\right)\right\}$. Le groupe endoscopique elliptique $H=U(1)\left(F_{0}\right) \times U(1)\left(F_{0}\right)$ de $G$ s'identifie au sous-groupe des éléments de $G$ dont la matrice dans la base orthogonale $\mathcal{B}^{o}=\left(e_{-1}+\frac{1}{2} e_{1}, e_{-1}-\frac{1}{2} e_{1}\right)$ est diagonale. A conjugaison près dans $G$, il existe au plus deux plongements de $H$. Ils sont alors conjugués par $g_{0}$.

Les définitions de l'application de transfert de $H$ à $G$ (cf. prop. 3.1) et du changement de base labile [18, §4.7] dépendent du choix d'un caractère de $F^{\times}$prolongeant $\omega_{F / F_{0}}$. On choisit le caractère $\mu$ défini précédemment. On a alors [18, §11.4] : si $\pi$ est une représentation admissible de $G$, son image par le changement de base stable est $\tilde{\pi}$ si et seulement si son image par le changement de base labile est $\tilde{\pi} \cdot \mu \circ$ dét. 
Pour établir le changement de base stable des paquets supercuspidaux singletons de $G$, on vérifie l'identité de caractères qui le caractérise et qui s'exprime grâce à la norme cyclique, notée $\mathcal{N}_{\tau}$ (4.6.1). Il s'agit d'une bijection de l'ensemble des classes de $\tau$-conjugaison stable de $\widetilde{G}$ dans l'ensemble des classes de conjugaison stable de $G$, qui associe à la classe de $\tau$-conjugaison stable de $g$, l'intersection de la classe de $\widetilde{G}$-conjugaison de $N_{\tau}(g)=g \tau(g)$ avec $G$ [14].

Rappelons que la classe de $\tau$-conjugaison d'un élément $g$ de $\widetilde{G}, \mathcal{C} l_{\tau}(g)$, est l'ensemble des éléments de $\widetilde{G}$ de la forme $h^{-1} g \tau(h)$ avec $h \in \widetilde{G}$ et que sa classe de $\tau$-conjugaison stable $\mathcal{C} l_{\tau}^{s t}(g)$ est l'ensemble des éléments $g^{\prime}$ de $\widetilde{G}$ tel que $N_{\tau}\left(g^{\prime}\right)$ soit $\widetilde{G}$-conjugué à $N_{\tau}(g)$.

De façon analogue, pour un élément $x$ de $G$, on désigne par $\mathcal{C} l(x)$ sa classe de conjugaison et par $\mathcal{C} l^{s t}(x)$ sa classe de conjugaison stable (c'est-à-dire de conjugaison sous $\widetilde{G}$ ).

\section{Les paquets supercuspidaux de $U(1,1)\left(F_{0}\right)$.}

Le groupe $G U(1,1)\left(F_{0}\right)$ agit par conjugaison sur l'ensemble des représentations lisses irréductibles de $U(1,1)\left(F_{0}\right)$. Les orbites sont exactement les paquets de $U(1,1)\left(F_{0}\right)[18, \S 11.1]$. On en déduit :

\section{Proposition.}

(i) Les représentations irréductibles très cuspidales de $U(1,1)\left(F_{0}\right)$ de niveau strictement positif sont seules dans leur paquet. Les autres représentations cuspidales de niveau strictement positif appartiennent à des paquets de cardinal 2.

(ii) Si $F$ est non ramifiée sur $F_{0}$, tous les paquets formés par des représentations irréductibles cuspidales de niveau 0 sont de cardinal 2.

(iii) Si $F$ est ramifiée sur $F_{0}$, tous les paquets formés par des représentations irréductibles cuspidales de niveau 0 sont de cardinal 1 sauf un. Ce dernier est $\left\{\operatorname{Ind}_{K}^{G} \sigma^{+}, \operatorname{Ind}_{K}^{G} \sigma^{-}\right\}$où $K$ est le sous-groupe parahorique maximal et $\sigma^{ \pm}$ les deux représentations de $S L_{2}\left(k_{0}\right)$ de dimension $\frac{q-1}{2}$ [19].

La suite du paragraphe justifie ces assertions.

Soient $\pi$ une représentation irréductible cuspidale de $G$ et $(J, \lambda)$ un type pour cette représentation : $\pi=\operatorname{Ind}_{J}^{G} \lambda$. Il suffit d'étudier si $\pi$ et ${ }^{g_{0}} \pi$, sa conjuguée par $g_{0}$, sont isomorphes. On distingue deux cas selon le niveau.

2.1. $\pi$ est de niveau strictement positif. Alors $(J, \lambda)$ provient d'une strate gauche semi-simple $\mathfrak{s}=\left(\mathcal{L}, n,\left[\frac{n}{2}\right], b\right)$ (au sens de [20, déf. 4.9]). On note $Z_{\widetilde{G}}(b)$ le centralisateur de $b$ dans $\widetilde{G}$ et $Z_{G}(b)$ celui de $b$ dans $G$ : $Z_{G}(b)=Z_{\widetilde{G}}(b) \cap G$.

Si $\pi$ est très cuspidale [3, $\S$ A.5], $Z_{\widetilde{G}}(b) \cup\{0\}$ est l'extension quadratique

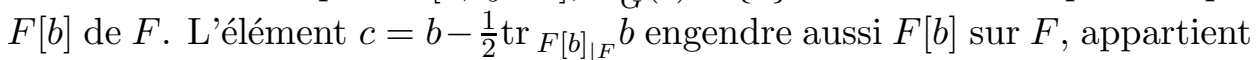
à l'algèbre de Lie de $G$ et engendre une extension quadratique $F_{0}[c]$ sur $F_{0}$, autre que $F$. On vérifie que les éléments de $F_{0}[c]^{\times}$sont des éléments de $G U(1,1)\left(F_{0}\right)$ dont le rapport est égal à leur norme dans $F_{0}$. Il en existe donc un, $c_{0}$, qui appartient à $g_{0} F_{0}^{\times} U(1,1)\left(F_{0}\right)$. Alors : ${ }^{g_{0}} \pi \simeq{ }^{c_{0}} \pi \simeq \pi$. 
Si $\pi$ est cuspidale scindée [3, $\S$ A.6], $Z_{\widetilde{G}}(b)$ est isomorphe à $F^{\times} \times F^{\times}$. On montre par l'absurde que l'entrelacement $\mathcal{E}\left(\mathfrak{s},{ }^{g_{0}} \mathfrak{s}\right)$ des strates semi-simples $\mathfrak{s}$ et ${ }^{g_{0}} \mathfrak{s}=\left(g_{0} \mathcal{L}, n,\left[\frac{n}{2}\right], g_{0}^{-1} b g_{0}\right)$ contenues dans $\lambda$ et ${ }^{g_{0}} \lambda$ respectivement, est vide (d'où l'on déduit que $\lambda$ et ${ }^{g_{0}} \lambda$ ne sont pas entrelacées dans $G$ ).

L'entrelacement $\widetilde{\mathcal{E}}\left(\mathfrak{s},{ }^{g_{0}} \mathfrak{s}\right)$ de $\mathfrak{s}$ et ${ }^{g_{0}} \mathfrak{s}$ dans $\widetilde{G}$ est égal à $\widetilde{\mathcal{E}}(\mathfrak{s}) g_{0}^{-1}$ où $\widetilde{\mathcal{E}}(\mathfrak{s})$ est l'entrelacement de $\mathfrak{s}$ dans $\widetilde{G}$. De plus, par le théorème 4.10 précisé par (4.15) de [20], $\widetilde{\mathcal{E}}(\mathfrak{s})$ est égal à $\widetilde{U}_{m}(\mathcal{L}) Z_{\widetilde{G}}(b) \widetilde{U}_{m}(\mathcal{L})$ avec $m=\left[\frac{n+1}{2}\right]$. On déduit que :

$$
\begin{aligned}
\mathcal{E}\left(\mathfrak{s},{ }^{g_{0}} \mathfrak{s}\right) & =\widetilde{\mathcal{E}}\left(\mathfrak{s},{ }^{g_{0}} \mathfrak{s}\right)^{\tau}=\left(\widetilde{U}_{m}(\mathcal{L}) Z_{\widetilde{G}}(b) g_{0}^{-1} \widetilde{U}_{m}\left(g_{0} \mathcal{L}\right)\right)^{\tau} \\
& =\cup_{z \in Z_{\widetilde{G}}(b)}\left(\widetilde{U}_{m}(\mathcal{L}) z g_{0}^{-1} \widetilde{U}_{m}\left(g_{0} \mathcal{L}\right)\right)^{\tau} .
\end{aligned}
$$

Si $\mathcal{E}\left(\mathfrak{s},{ }^{g_{0}} \mathfrak{s}\right) \neq \emptyset$, il existe $z \in Z_{\widetilde{G}}(b)$ tel que : $\left(\widetilde{U}_{m}(\mathcal{L}) z g_{0}^{-1} \widetilde{U}_{m}\left(g_{0} \mathcal{L}\right)\right)^{\tau} \neq \emptyset$. Fixons un tel $z$. Comme $\mathcal{L}$ et $g_{0} \mathcal{L}$ sont des chaînes autoduales $\left(g_{0}\right.$ appartient à $\left.G U(1,1)\left(F_{0}\right)\right)$, les deux pro-p-sous-groupes $\widetilde{U}_{m}(\mathcal{L})$ et $\widetilde{U}_{m}\left(g_{0} \mathcal{L}\right)$ sont invariants par $\tau$. Par le lemme 2.2 de [20], généralisé sans encombre au cas de deux pro-p-groupes, $\widetilde{U}_{m}(\mathcal{L}) z g_{0}^{-1} \widetilde{U}_{m}\left(g_{0} \mathcal{L}\right)$ est invariant par $\tau$. En particulier $\tau\left(z g_{0}^{-1}\right)$, qui est égal à $\alpha_{0} \tau(z) g_{0}^{-1}$, appartient à $\widetilde{U}_{m}(\mathcal{L}) z g_{0}^{-1} \widetilde{U}_{m}\left(g_{0} \mathcal{L}\right)=$ $\widetilde{U}_{m}(\mathcal{L}) z \widetilde{U}_{m}(\mathcal{L}) g_{0}^{-1}$ d'où : $\alpha_{0} z^{-1} \tau(z)=z^{-1} u z \cdot u^{\prime}$ avec $u, u^{\prime} \in \widetilde{U}_{m}(\mathcal{L})$.

Exprimés dans une base orthogonale formée de vecteurs propres de $b$ et adaptée à $\mathcal{L}$, le terme de gauche est une matrice diagonale dont les termes diagonaux appartiennent à $\alpha_{0} N_{F / F_{0}}\left(F^{\times}\right)$tandis que le terme de droite a au moins un de ses coefficients diagonaux dans $1+\mathfrak{p}^{m}$. L'égalité entraîne donc que $\alpha_{0}$ doit être une norme de $F^{\times}$dans $F_{0}$ ce qui est absurde.

2.2. $\pi$ est de niveau 0 . Il existe un sous-groupe parahorique $K$ maximal (au sens de Bruhat-Tits) et une représentation irréductible $\sigma$ de $K$, triviale sur le radical pro-unipotent $K_{1}$ et définissant une représentation cuspidale du quotient réductif (connexe) $K / K_{1}$, tels que $\pi$ soit isomorphe à $\operatorname{Ind}_{K}^{G} \sigma$ (dans ce cas, $K$ est son propre normalisateur) [17, $\S \S 2$ et 3$]$.

Quand $F$ est non ramifiée sur $F_{0}$, il existe, à conjugaison près, deux sousgroupes parahoriques maximaux : $K=U_{0}(\mathcal{L})$ où $\mathcal{L}$ est une chaîne de réseaux autoduale de période 1 , d'invariant pair ou impair, et $K^{\prime}$ son conjugué par $g_{0}$. Dans ce cas, $\pi=\operatorname{Ind}_{K}^{G} \sigma$ et ${ }^{g_{0}} \pi=\operatorname{Ind}_{K^{\prime}}^{G}{ }^{g_{0}} \sigma$ ne sont pas isomorphes.

En effet, puisque $U(1,1)\left(F_{0}\right)=K S U(1,1)\left(F_{0}\right)$, les restrictions à $S U(1,1)\left(F_{0}\right)$ de $\pi$ et ${ }^{g_{0}} \pi$ sont deux représentations irréductibles de $S U(1,1)\left(F_{0}\right)$ dont les conjuguées par $\varepsilon$, notées ${ }^{\varepsilon} \pi$ et ${ }^{\varepsilon}\left({ }^{g_{0}} \pi\right)$ respectivement, sont deux représentations de $S L_{2}\left(F_{0}\right)$, cuspidales, irréductibles et de niveau $0:{ }^{\varepsilon} \pi=\operatorname{Ind}_{\varepsilon_{K}}^{S L_{2}\left(F_{0}\right)_{\varepsilon}} \sigma$ et ${ }^{\varepsilon}\left(g_{0} \pi\right)=g_{0}\left({ }^{\varepsilon} \pi\right)$ (puisque $\varepsilon$ et $g_{0}$ commutent). Elles sont donc toutes deux sous-représentations de la restriction à $S L_{2}\left(F_{0}\right)$ d'une représentation irréductible cuspidale de niveau 0 de $G L_{2}\left(F_{0}\right)$ et ne sont donc pas équivalentes [15, th. 4.4].

Lorsque $F$ est ramifiée, il existe une unique classe de conjugaison de sous-groupes parahoriques maximaux représentée par $U_{0}(\mathcal{L})$ où $\mathcal{L}$ est la chaîne de réseaux autoduale de période 1 et d'invariant impair. Le quotient $U_{0}(\mathcal{L}) / U_{1}(\mathcal{L})$ est isomorphe à $S L_{2}\left(k_{0}\right)$.

Ainsi, $\sigma$ définit une représentation cuspidale irréductible de $S L_{2}\left(k_{0}\right)$. D'après T. A. Springer [19, II §3], $\sigma$ est de dimension $q-1$ ou $\frac{q-1}{2}$ et est décrite par 
son caractère. Il est alors aisé de montrer que si $\sigma$ est de dimension $q-1$, elle est isomorphe à sa conjuguée par $g_{0}$ tandis que si sa dimension est $\frac{q-1}{2}$, sa conjuguée est l'autre représentation cuspidale de même dimension, notée $\sigma^{\prime}$. Dans le premier cas, $\pi$ est isomorphe à sa conjuguée par $g_{0}$. Dans le deuxième cas, montrons que $\sigma$ et $\sigma^{\prime}$ ne sont pas entrelacées.

Grâce à la décomposition de Cartan, $K$ étant un bon compact dans ce cas, il suffit de montrer que $g=\left(\begin{array}{cc}\varpi & 0 \\ 0 & \bar{\varpi}^{-1}\end{array}\right)^{m}, m \in \mathbb{N}$, n'entrelace pas $\sigma$ et $\sigma^{\prime}$. Si $m=0$, c'est clair car $\sigma$ et $\sigma^{\prime}$ ne sont pas isomorphes. Si $m>0$, $K \cap{ }^{g} K$ contient le sous-groupe $\left(\begin{array}{cc}1 & 0 \\ \varpi \mathfrak{o}_{0} & 1\end{array}\right)$. Or la restriction de $\sigma^{\prime}$ à ce sous-groupe ne contient pas le caractère trivial tandis que celle de ${ }^{g} \sigma$ est triviale.

\section{Les paquets endoscopiques CUSPidaux et les CARACtères De $H$.}

Il s'agit d'identifier le caractère $\theta$ de $H$ associé à un paquet endoscopique cuspidal de $G$ par l'application de transfert.

Remarquons que lorsque $F$ est ramifiée sur $F_{0}$, il existe un unique paquet endoscopique de niveau 0 et deux caractères réguliers de $H$ de niveau 0 : $\theta=1 \otimes \chi$ et $\chi \otimes 1={ }^{\tau} \theta$ où $\chi$ est le caractère de $F_{\mid F_{0}}^{1}$ d'ordre 2 trivial sur $(1+\mathfrak{p}){ }_{\mid F_{0}}^{1}$. Nécessairement, le paquet endoscopique est l'image de $\theta$ (et ${ }^{\tau} \theta$ ) par l'application de transfert. Par la suite, on suppose donc :

$$
n>0 \quad \text { ou } \quad F \text { n'est pas ramifiée sur } F_{0} \text {. }
$$

3.1. Soit $\Pi$ un paquet endoscopique cuspidal de $G$. Il est entièrement déterminé par la donnée d'un type simple $(J, \lambda)$ :

(a) $J=H U_{m}(\mathcal{L})$ où $\mathcal{L}$ est la chaîne de $\mathfrak{o}$-réseaux autoduale stable par $H$, c'est-à-dire celle de période 1 , d'invariant pair, et $m=\left[\frac{n+1}{2}\right]$, $n \in \mathbb{N}$;

(b) Cas de niveau $0, F$ non ramifiée : $\lambda$ est le relèvement à $U_{0}(\mathcal{L})$ d'une représentation irréductible cuspidale $\sigma$ de $U(1,1)\left(k_{0}\right)$ vérifiant :

$$
\forall x \in H\left(k_{0}\right), \operatorname{tr} \sigma(x)= \begin{cases}(q-1) \cdot \chi(x) & \text { si } x \in Z\left(k_{0}\right) \\ -\left(\chi(x)+\chi\left(w x w^{-1}\right)\right) & \text { si } x \notin Z\left(k_{0}\right)\end{cases}
$$

pour un caractère régulier $\chi$ de $H\left(k_{0}\right)[8, \S 6]$. L'élément $w$ appartient au normalisateur de $H\left(k_{0}\right)$ mais non à $H\left(k_{0}\right)$. Notons $\theta_{1} \otimes \theta_{2}$ le caractère de $H$ relevant $\chi$.

(b') Cas de niveau strictement positif: $\lambda$ est une représentation irréductible de $J$ dont la restriction à $U_{m}(\mathcal{L})$ est multiple d'un caractère $\psi_{b}$ avec $b \in \mathfrak{a}_{-n}(\mathcal{L})^{-} \backslash \mathfrak{a}_{-n+1}(\mathcal{L})^{-}$et dont la restriction à $H$ vérifie :

$$
\forall x \in H, \operatorname{tr} \lambda(x)= \begin{cases}\operatorname{dim} \lambda \cdot \theta_{1} \otimes \theta_{2}(x) & \text { si } x \in Z\left(F_{0}\right) \\ -\theta_{1} \otimes \theta_{2}(x) & \text { si } x \notin Z\left(F_{0}\right)\end{cases}
$$

pour un caractère régulier $\theta_{1} \otimes \theta_{2}$ de $H$ prolongeant $\psi_{b \mid H \cap U_{m}(\mathcal{L})}$ (voir $\$ 5$ et en particulier $\$ 5.3 .4$. (1)). Précisons que $b$ est de la forme $b_{1} \oplus b_{2}$ dans la décomposition de $F^{2}$ définie par $\mathcal{B}^{o}$ avec $b_{1}-b_{2} \notin \mathfrak{p}^{-n+1}$ (quitte à tordre $\Pi$ par un caractère de $G$ ).

On a alors : $\Pi=\left\{\pi,{ }^{g_{0}} \pi\right\}$ où $\pi=\operatorname{Ind}_{J}^{G} \lambda$. 
Proposition $([18])$. Soit $\theta$ le caractère de $H$ correspondant à $\Pi$ par l'application de transfert. Il existe un unique $\epsilon_{\theta} \in\{ \pm 1\}$ tel que pour tout $h \in H_{G-r e g}$, $h=\left(h_{1}, h_{2}\right)$ (c'est-à-dire $h$ de matrice $\operatorname{diag}\left(h_{1}, h_{2}\right)$ dans $\left.\mathcal{B}^{o}\right)$,

$$
\operatorname{tr} \pi(h)-\operatorname{tr}^{g_{0}} \pi(h)=\epsilon_{\theta} \frac{\iota(h)}{D_{G}(h)}\left(\theta(h)+\theta\left(w h w^{-1}\right)\right)
$$

où $\iota(h)=\mu\left(h_{1}-h_{2}\right), D_{G}(h)=\left|\frac{\left(h_{1}-h_{2}\right)^{2}}{h_{1} h_{2}}\right|_{F_{0}}^{\frac{1}{2}}$ et $w=\left(\begin{array}{ll}0 & 1 \\ 1 & 0\end{array}\right)$ dans $\mathcal{B}^{o}$.

3.2. Evaluons le membre de gauche de (3.1.1) en suivant la méthode de Y. Flicker [9], c'est-à-dire en se ramenant aux résultats de [16, §2], grâce à l'isomorphisme $\Phi$ entre $G Z(F)$ et $G L\left(2, F_{0}\right)^{+} Z(F)$ (cf. \$1). Précisons que dorénavant, toutes les matrices sont exprimées relativement à la base $\mathcal{B}$.

On note $i$ le plongement de $F^{\times}$dans $G L\left(2, F_{0}\right)^{+}$qui à $x=\alpha+\varepsilon \beta \in F^{\times}$ $\left(\alpha, \beta \in F_{0}\right)$ associe $i(x)=\left(\begin{array}{cc}\alpha & 2 \beta \\ \frac{\varepsilon^{2}}{2} \beta & \alpha\end{array}\right)$.

\section{Lemme.}

(i) Soient $h=\left(h_{1}, h_{2}\right) \in H, x \in F^{\times}$et $z \in Z(F)$. Alors :

$$
\begin{array}{ll} 
& \Phi(h z)=i\left(\bar{a} h_{1}\right) \cdot \bar{a}^{-1} z \text { où } a \in F^{\times} \text {tel que } a \bar{a}^{-1}=\text { dét } h=h_{1} h_{2} \\
\text { et } \quad & \Phi^{-1}(x z)=h_{x} \cdot \bar{x} z \text { où } h_{x}=\left(x \bar{x}^{-1}, 1\right) \in H .
\end{array}
$$

En particulier, $\Phi$ identifie $H Z(F)$ et $i\left(F^{\times}\right) Z(F)$.

(ii) Soient $k \in \mathbb{N}$ et $\mathcal{L}^{0}$ la chaîne de $\mathfrak{o}_{0}$-réseaux dans $F_{0}^{2}$ stable par $F^{\times}$. Lorsque $k=0$, on suppose en outre que $F$ n'est pas ramifiée sur $F_{0}$. Alors : $\Phi\left(U_{k}(\mathcal{L}) Z\left(F_{0}\right)\right)=\left\{u z, u \in U_{k}\left(\mathcal{L}^{0}\right), z \in Z(F)\right.$ tels que détu $\left.\cdot N_{F_{\mid F_{0}}}(z)=1\right\}$. (iii) $\Phi(J Z(F)) \cap G L\left(2, F_{0}\right)^{+}=i\left(F^{\times}\right) U_{m}\left(\mathcal{L}^{0}\right)$.

Démonstration. L'assertion (i) est obtenue par de simples calculs tandis que (iii) est une conséquence immédiate de (i) et (ii).

(ii) Supposons d'abord $k>0$. Soient $x \in U_{k}(\mathcal{L})$ et $z \in Z\left(F_{0}\right)$. Il existe $y \in 1+\mathfrak{p}^{k}$ tel que : $y \bar{y}^{-1}=$ dét $x$, puis $x^{\prime} \in U_{k}(\mathcal{L}) \cap S U(1,1)\left(F_{0}\right)$ tel que : $x=\left(\begin{array}{cc}y & 0 \\ 0 & \bar{y}^{-1}\end{array}\right) x^{\prime}$. Alors : $\Phi(x z)=\left(\begin{array}{cc}y \bar{y} & 0 \\ 0 & 1\end{array}\right) \Phi\left(x^{\prime}\right) \cdot \bar{y} z$.

Or $x^{\prime}$ est de la forme $\left(\begin{array}{cc}a & \varepsilon b \\ \varepsilon^{-1} c & d\end{array}\right)$ avec $a, d \in\left(1+\mathfrak{p}^{k}\right) \cap F_{0}=1+\mathfrak{p}_{0}^{\left[\frac{k+1}{e_{0}}\right]}$ et $\varepsilon b, \varepsilon^{-1} c \in \mathfrak{p}^{k} \cap F_{0}=\mathfrak{p}_{0}^{\left[\frac{k}{e_{0}}\right]}$ donc $\Phi\left(x^{\prime}\right)$ appartient à

$$
\left\{\begin{array}{r}
\left(\begin{array}{cc}
1+\mathfrak{p}_{0}^{k} & \mathfrak{p}_{0}^{k} \\
\mathfrak{p}_{0}^{k} & 1+\mathfrak{p}_{0}^{k}
\end{array}\right) \cap S L(2)\left(F_{0}\right) \subset U_{k}\left(\mathcal{L}^{0}\right) \text { si } e_{0}=1 \\
\left(\begin{array}{cc}
1+\mathfrak{p}_{0}^{\left[\frac{k+1}{2}\right]} & \mathfrak{p}_{0}^{\left[\frac{k}{2}\right]} \\
\mathfrak{p}_{0}^{\left[\frac{k}{2}\right]+1} & 1+\mathfrak{p}_{0}^{\left[\frac{k+1}{2}\right]}
\end{array}\right) \cap S L(2)\left(F_{0}\right) \subset U_{k}\left(\mathcal{L}^{0}\right) \text { si } e_{0}=2 .
\end{array}\right.
$$

De plus, $y \bar{y} \in 1+\operatorname{tr} \mathfrak{p}^{k}$ donc $\left(\begin{array}{cc}y \bar{y} & 0 \\ 0 & 1\end{array}\right) \in U_{k}\left(\mathcal{L}^{0}\right)$. Ainsi, $\Phi(x)$ appartient à $U_{k}\left(\mathcal{L}^{0}\right) Z(F)$ et dét $\left(\left(\begin{array}{cc}y \bar{y} & 0 \\ 0 & 1\end{array}\right) \Phi\left(x^{\prime}\right)\right) \cdot N_{F_{\mid F_{0}}}\left(\bar{y}^{-1} z\right)=1$.

L'inclusion inverse se montre de façon analogue. 
Lorsque $k$ est nul, $F$ est non ramifiée sur $F_{0}$ : les mêmes arguments fournissent les mêmes résultats.

3.3. Pour poursuivre, on choisit un caractère $\Omega$ de $Z(F)$ prolongeant le caractère central $\omega_{\pi}$ de $\pi$ et tel que

$$
\left\{\begin{array}{l}
\forall u \in \mathfrak{a}_{\left[\frac{n}{2}\right]+1}(\mathcal{L}) \cap Z(F), \quad \Omega(1+u)=\psi\left(\left(b_{1}+b_{2}\right) u\right) \text { si } n>0 \\
\Omega=\Theta_{1} \Theta_{2} \text { où } \Theta_{i} \text { est un prolongement de } \theta_{i} \text { à } F^{\times} \text {si } n=0 .
\end{array}\right.
$$

On prolonge alors $\pi$ et ${ }^{g_{0}} \pi$ à $G Z(F)$ en faisant agir $Z(F)$ via $\Omega$. On note encore $\pi$ et ${ }^{g_{0}} \pi$ ces prolongements puis :

$$
\pi_{0}^{+}=\pi \circ \Phi_{\mid G L_{2}\left(F_{0}\right)^{+}}^{-1}, \quad \pi_{0}^{-}={ }^{g_{0}} \pi \circ \Phi_{\mid G L_{2}\left(F_{0}\right)^{+}}^{-1}={ }^{g_{0}} \pi_{0}^{+}, \quad \pi_{0}=\operatorname{Ind}_{G L_{2}\left(F_{0}\right)^{+}}^{G L_{2}\left(F_{0}\right)} \pi_{0}^{+} .
$$

Lemme. Posons : $J_{0}=F^{\times} U_{m}\left(\mathcal{L}^{0}\right), J_{0, c}=\mathfrak{o}_{0}^{\times} U_{m}\left(\mathcal{L}^{0}\right)$. Notons $\Lambda$ la représentation $(\lambda \Omega) \circ \Phi_{\mid J_{0}}^{-1}$ de $J_{0}$ et $\Lambda_{c}$ sa restriction $\grave{a} J_{0, c}$.

(i) La paire $\left(J_{0, c}, \Lambda_{c}\right)$ est un type simple maximal de $\pi_{0}$ et $\pi_{0}=\operatorname{Ind}_{J_{0}}^{G L_{2}\left(F_{0}\right)} \Lambda$. La représentation $\pi_{0}$ est donc irréductible et cuspidale.

(ii) Cas $n>0$. La restriction de $\Lambda$ à $U_{\left[\frac{n}{2}\right]+1}\left(\mathcal{L}^{0}\right)$ est un multiple du caractère $\psi_{0, \alpha}$ où $\alpha=i\left(b_{1}-b_{2}\right) \in \mathfrak{a}_{-n}\left(\mathcal{L}^{0}\right) \backslash \mathfrak{a}_{-n+1}\left(\mathcal{L}^{0}\right)$.

La restriction de $\Lambda$ à $F^{\times}$vérifie pour tout $x \in F^{\times}$:

$$
\begin{array}{ll}
\text { si } \operatorname{dim} \Lambda=1, & \Lambda(x)=\widetilde{\theta}_{1}(x) \Omega(\bar{x}) ; \\
\text { si } \operatorname{dim} \Lambda>1, & \operatorname{tr} \Lambda(x)=\left\{\begin{array}{ll}
\operatorname{dim} \Lambda \cdot \widetilde{\theta}_{1}(x) \Omega(\bar{x}) & \text { si } x \in F_{0}^{\times} \\
-\widetilde{\theta}_{1}(x) \Omega(\bar{x}) & \text { sinon. }
\end{array} .\right.
\end{array}
$$

(iii) Cas $n=0$ (F n'est pas ramifiée). La restriction de $\Lambda$ à $U_{0}\left(\mathcal{L}^{0}\right)$ est le relèvement d'une représentation $\sigma_{0}$ de $G L_{2}\left(k_{0}\right)$ caractérisée par :

$$
\left\{\begin{aligned}
\operatorname{tr} \sigma_{0}(z) & =(q-1) \Omega(z), \quad z \in k_{0}^{\times} \\
\operatorname{tr} \sigma_{0}(z n) & =-\Omega(z), \quad z \in k_{0}^{\times}, n \in N\left(k_{0}\right)-\{\mathrm{id}\} \\
\operatorname{tr} \sigma_{0}(x) & =-\Omega(\bar{x})\left(\widetilde{\theta}_{1}(x)+\widetilde{\theta}_{2}(x)\right), \quad x \in k^{\times}-k_{0}^{\times}
\end{aligned}\right.
$$

et $\Lambda\left(\varpi_{0}\right)=\Omega\left(\varpi_{0}\right)$.

Démonstration. La première assertion est une conséquence des deux suivantes et ces dernières proviennent des propriétés de $\lambda$ et de $\Omega$ via $\Phi$.

Plus précisément, pour établir (ii), on étudie d'abord la restriction de $\Lambda$ à $U_{\left[\frac{n}{2}\right]+1}\left(\mathcal{L}^{0}\right) \cap S U(1,1)\left(F_{0}\right)$. Un calcul du même style que dans le lemme 3.2 montre que cette restriction est multiple de $\psi_{0, \alpha}$ où $\alpha=i\left(b_{1}-b_{2}\right)+D$ avec $D$ une matrice diagonale. Ensuite, si $x=\operatorname{diag}\left(z, \bar{z}^{-1}\right) \in U_{\left[\frac{n}{2}\right]+1}\left(\mathcal{L}^{0}\right), \Lambda(x)$ est multiple de $\psi_{\varepsilon_{b}}(x) \Omega(\bar{z})$, qui vaut 1 par choix de $\Omega$ (3.3.1). On peut donc prendre $D=0$. L'élément $\alpha$ ainsi défini appartient à $\mathfrak{a}_{-n}\left(\mathcal{L}^{0}\right) \backslash \mathfrak{a}_{-n+1}\left(\mathcal{L}^{0}\right)$ par 3.1 (b'). La suite est immédiate, tout comme l'assertion (iii).

Corollaire. Soit $\Delta_{\theta_{1} \otimes \theta_{2}}$ le caractère de $F^{\times}$de niveau 0 défini par :

(i) quand $F$ n'est pas ramifiée sur $F_{0}, \Delta_{\theta_{1} \otimes \theta_{2}}=\mu$;

(ii) quand $F$ est ramifiée sur $F_{0}$,

$$
\Delta_{\theta_{1} \otimes \theta_{2} \mid F_{0} \times}=\omega_{F / F_{0}} \text { et } \Delta_{\theta_{1} \otimes \theta_{2}}(\varpi)=\omega_{F / F_{0}}\left(\varpi\left(b_{1}-b_{2}\right)\right) \lambda_{F_{\mid F_{0}}}\left(\psi_{0}\right)^{-1} \text {. }
$$

Posons : $\Theta=\Delta_{\theta_{1} \otimes \theta_{2}}^{-1} \widetilde{\theta}_{1} \bar{\Omega}$ si $n>0$ et $\Theta=\mu^{-1} \Theta_{1} \bar{\Theta}_{2}$ si $n=0$. Alors $\pi_{0}$ est la représentation de $G L_{2}\left(F_{0}\right)$ associée au caractère $\Theta$ par [13]. 
Démonstration. C'est une application des résultats de [7], $\S \S 19$ et 34 .

3.4. D'après la théorie de Mackey, la restriction de $\pi_{0}$ à $G L_{2}\left(F_{0}\right)^{+}$est la somme des représentations $\pi_{0}^{+}$et $\pi_{0}^{-}$. D'après [16, p.738], pour tout $x \in F^{\times}$ régulier,

$$
\operatorname{tr} \pi_{0}^{+}(x)-\operatorname{tr} \pi_{0}^{-}(x)= \pm \lambda_{F_{\mid F_{0}}}\left(\psi_{0}\right) \omega_{F / F_{0}}\left(\frac{x-\bar{x}}{\varepsilon}\right) \frac{\Theta(x)+\Theta(\bar{x})}{D(x)},
$$

où $D(x)=\left|\frac{(x-\bar{x})^{2}}{x \bar{x}}\right|_{F_{0}}^{1 / 2}$. Mais, si $h=\left(h_{1}, h_{2}\right) \in H$ est $G$-régulier et $a \in F^{\times}$ tel que $a \bar{a}=h_{1} h_{2}, \bar{a} h_{1}$ est un élément de $F^{\times}$régulier dont le conjugué est $\bar{a} h_{2}$ et l'on a :

$$
\begin{aligned}
& \operatorname{tr} \pi(h)=\operatorname{tr} \pi\left(\Phi^{-1}\left(i\left(\bar{a} h_{1}\right) \cdot \bar{a}^{-1}\right)\right)=\operatorname{tr} \pi_{0}^{+}\left(i\left(\bar{a} h_{1}\right)\right) \Omega\left(\bar{a}^{-1}\right), \\
& \text { et } \quad \operatorname{tr}^{g_{0}} \pi(h)=\operatorname{tr} \pi_{0}^{-}\left(i\left(\bar{a} h_{1}\right)\right) \Omega\left(\bar{a}^{-1}\right) .
\end{aligned}
$$

Par suite (on note $\Delta=\Delta_{\theta_{1} \otimes \theta_{2}}$ ):

$$
\begin{aligned}
& \operatorname{tr} \pi(h)-\operatorname{tr}^{g_{0}} \pi(h)= \pm \lambda_{F_{\mid F_{0}}}\left(\psi_{0}\right) \omega_{F / F_{0}}\left(\frac{\bar{a} h_{1}-\bar{a} h_{2}}{\varepsilon}\right) \Omega\left(\bar{a}^{-1}\right) \frac{\Theta\left(\bar{a} h_{1}\right)+\Theta\left(\bar{a} h_{2}\right)}{\Delta\left(\bar{a} h_{1}\right)} \\
&= \pm \lambda_{F_{\mid F_{0}}}\left(\psi_{0}\right) \mu\left(h_{1}-h_{2}\right) \mu\left(\frac{\bar{a}}{\varepsilon}\right) \Delta^{-1}(\bar{a}) \Omega\left(\bar{a}^{-1}\right) \\
& \cdot \frac{\Delta^{-1}\left(h_{1}\right) \widetilde{\theta}_{1}\left(\bar{a} h_{1}\right) \Omega\left(\bar{a} h_{2}\right)+\Delta^{-1}\left(h_{2}\right) \widetilde{\theta}_{1}\left(\bar{a} h_{2}\right) \Omega\left(\bar{a} h_{1}\right)}{D_{G}(h)} \\
&= \pm \lambda_{F_{\mid F_{0}}}\left(\psi_{0}\right) \mu(\varepsilon)^{-1} \frac{\iota(h)}{D_{G}(h)} \mu(\bar{a}) \Delta^{-1}(\bar{a}) \\
& \cdot\left(\mu^{-1} \theta_{1} \otimes \theta_{2}(h)+\mu^{-1} \theta_{1} \otimes \theta_{2}\left(w h w^{-1}\right)\right)
\end{aligned}
$$

en remarquant que : $\Delta(x)=\mu(x)$ pour tout $x \in F_{\mid F_{0}}^{1}$ et $\widetilde{\theta}_{1}\left(\bar{a} h_{1}\right)=\theta_{1}\left(h_{1} h_{2}^{-1}\right)$ tandis que $\Omega\left(\bar{a}^{-1}\right) \Omega\left(\bar{a} h_{2}\right)=\Omega\left(h_{1} h_{2}\right) \Omega\left(h_{1}^{-1}\right)=\theta_{1}\left(h_{2}\right) \theta_{2}\left(h_{2}\right)$ car $h_{2} \in F_{\mid F_{0}}^{1}$. De plus, $\lambda_{F_{\mid F_{0}}}\left(\psi_{0}\right) \mu(\varepsilon)^{-1}$ est égal à -1 si $F$ est non ramifiée et à 1 si $F$ est ramifiée (par choix de $\varepsilon$ et $\mu$ ) et le caractère de $F^{\times}, a \mapsto \mu(\bar{a}) \Delta^{-1}(\bar{a})$, est trivial si $F$ n'est pas ramifiée et égal à $\left(\omega_{F / F_{0}}\left(\varpi\left(b_{1}-b_{2}\right)\right)^{\text {val }(a)}\right.$ si $F$ est ramifiée. Dans ce dernier cas, $a$ est de valuation paire si et seulement si $h_{1} h_{2} \in(1+\mathfrak{p})_{F_{0}}^{1}$. En comparant à (3.1.1), on conclut :

Proposition. Soit $\Pi=\left\{\pi,{ }^{g_{0}} \pi\right\}$ un paquet endoscopique cuspidal de $G$ décrit en 3.1 (dont on reprend les notations). On définit un caractère $\delta_{\theta_{1} \otimes \theta_{2}}$ de $F_{\mid F_{0}}^{1}$ par : $\delta_{\theta_{1} \otimes \theta_{2}}$ est trivial si $F$ n'est pas ramifiée sur $F_{0} ; \delta_{\theta_{1} \otimes \theta_{2}}$ est trivial sur $(1+\mathfrak{p})_{\mid F_{0}}^{1}$ et $\delta_{\theta_{1} \otimes \theta_{2}}(-1)=\omega_{F / F_{0}}\left(\varpi\left(b_{1}-b_{2}\right)\right)$ si $F$ est ramifiée.

Alors le paquet $\Pi$ est l'image par l'application de transfert du caractère $\theta$ de $H$ défini par :

$$
\forall h \in H, \quad \theta(h)=\delta_{\theta_{1} \otimes \theta_{2}}(\text { déth }) \cdot \mu^{-1} \theta_{1} \otimes \theta_{2}(h) .
$$

En application de la proposition 11.4.1(a) de [18], on obtient :

Corollaire. L'image par le changement de base stable du paquet $\Pi$ décrit en 3.1 (dont on reprend les notations) est $\widetilde{\pi}=\operatorname{ind}_{\widetilde{P}}^{G L_{2}(F)} \mu(\theta)$ où $\widetilde{P}$ est un sousgroupe parabolique de $G L_{2}(F)$ de facteur de Levi $H(F)$ et $\mu(\theta)$ le caractère 
de $H(F)$ défini par :

$$
\forall h \in H(F), \quad \mu(\theta)(h)=\mu^{-1} \widetilde{\delta}_{\theta_{1} \otimes \theta_{2}}(\text { dét } h) \cdot \widetilde{\mu}^{-1} \widetilde{\theta}_{1} \otimes \widetilde{\theta}_{2}(h) .
$$

Lorsque $F$ est ramifiée sur $F_{0}$ et que $\Pi$ est l'unique paquet endoscopique de niveau 0 , l'image de $\Pi$ est $\widetilde{\pi}=\operatorname{ind}_{\widetilde{P}}^{G L_{2}(F)} \mu^{-1} \otimes \widetilde{\chi} \mu^{-1}$.

\section{Changement de Base Stable Des Paquets Cuspidaux singletons.}

On procède en trois étapes : la première est consacrée à la construction de représentations irréductibles cuspidales de $\widetilde{G}=G L_{2}(F), \tau$-invariantes et de caractère central trivial sur $F_{0}^{\times}$à partir de représentations très cuspidales de G. Par [18, prop. 11.4.1(c)], ces représentations appartiennent à l'image d'un des deux changements de base, le "stable" ou le "labile". Dans la deuxième étape, on distingue parmi les représentations construites celles qui appartiennent à l'image du changement de base stable. La dernière étape décrit les changements de base stable et labile des paquets singletons de $G$.

4.1. Soit $(J, \lambda)$ un type simple maximal de $G$, c'est-à-dire :

(a) Cas de niveau 0 ( $F$ ramifiée sur $\left.F_{0}\right): J$ est le sous-groupe parahorique maximal de $G$, c'est-à-dire $J=U_{0}(\mathcal{L})$ avec $\mathcal{L}$ la chaîne autoduale de $F^{2}$, de période 1 et d'invariant impair. La représentation $\lambda$ est une représentation irréductible de $J$, triviale sur le sous-groupe pro-unipotent $J_{1}$ de $J$ et dont la factorisation $\bar{\lambda}$ par $J / J_{1} \simeq S L_{2}\left(k_{0}\right)$ est une représentation cuspidale de dimension $q-1$. Elle est associée à un caractère $\theta$ régulier d'ordre différent de 2 du groupe des éléments de norme 1 de l'extension quadratique $\ell$ de $k_{0}$ par :

$$
\begin{cases}\operatorname{tr} \bar{\lambda}(x)=(q-1) \theta(x) & \text { si } x \in\{ \pm 1\} \\ \operatorname{tr} \bar{\lambda}(x n)=-\theta(x) & \text { si } x \in\{ \pm 1\}, n \in N\left(k_{0}\right)-\{\text { id }\} \\ \operatorname{tr} \bar{\lambda}(x)=-(\theta(x)+\theta(\gamma(x))) & \text { si } x \in \ell_{\mid k_{0}}^{1}-\{ \pm 1\}\end{cases}
$$

où $\gamma$ est l'élément non trivial de $\mathcal{G} a l\left(\ell / k_{0}\right)[19$.

(b) Cas de niveau strictement positif: $(J, \lambda)$ provient d'une strate gauche très cuspidale $(\mathcal{L}, n, n-1, b)$ avec $n>0$, c'est-à-dire, en notant $E=F[b]$ l'extension de $F$ engendrée par $b$ et $L=E^{\sigma}$ la sousextension de $E$ formée des points fixes par $\sigma$,

$$
\begin{aligned}
J=\mathfrak{o}_{E \mid L}^{1} U_{\left[\frac{n+1}{2}\right]}(\mathcal{L}) \supset J_{1}=\left(1+\mathfrak{p}_{E}\right)_{\mid L}^{1} U_{\left[\frac{n+1}{2}\right]}(\mathcal{L}) \\
\supset H_{1}=\left(1+\mathfrak{p}_{E}\right)_{\mid L}^{1} U_{\left[\frac{n}{2}\right]+1}(\mathcal{L})
\end{aligned}
$$

et $\lambda$ est obtenue à partir d'un caractère $\theta$ de $H_{1}$ prolongeant le caractère $\psi_{b}$ de $U_{\left[\frac{n}{2}\right]+1}(\mathcal{L})$, comme un prolongement de l'unique représentation irréductible $\eta_{\theta}$ de $J_{1}$ contenant $\theta\left[3\right.$, annexe]. On note $\omega_{\lambda}$ le caractère central de $\lambda$.

On note $(\pi, \mathcal{V})$ l'induite compacte de $J$ à $G$ de $\lambda$. C'est une représentation irréductible très cuspidale de $G$ (et toute représentation irréductible très cuspidale de $G$ s'obtient ainsi). 
4.2. Construction dans le cas de niveau $\mathbf{0}$. On considère le type simple $(J, \lambda)$ décrit en 4.1 (a) (dont on reprend les notations) et on construit deux types simples maximaux $\tau$-invariants de $\widetilde{G},(\widetilde{J}, \widetilde{\Lambda})$ et $\left(\widetilde{J}, \widetilde{\Lambda}^{\prime}\right)$.

On choisit $\widetilde{J}=F^{\times} \widetilde{U}_{0}(\mathcal{L})=\varpi^{\mathbb{Z}} \widetilde{U}_{0}(\mathcal{L})$. La représentation $\widetilde{\Lambda}$ est un prolongement d'une représentation $\tau$-invariante $\widetilde{\lambda}$ de $\widetilde{U}_{0}(\mathcal{L})$ triviale sur $\widetilde{U}_{1}(\mathcal{L})$ qui se factorise en une représentation cuspidale $\overline{\widetilde{\lambda}}$ de $U_{0}(\mathcal{L}) / U_{1}(\mathcal{L}) \simeq G L_{2}\left(k_{0}\right)$. On définit donc $\widetilde{\lambda}$ comme suit.

On considère le caractère $\widetilde{\theta}$ de $\ell^{\times}$défini par : $\widetilde{\theta}(x)=\theta\left(x \underline{\gamma}(x)^{-1}\right), x \in \ell^{\times}$. Il est régulier donc associé à une représentation cuspidale $\overline{\widetilde{\lambda}}(\widetilde{\theta})$ de $G L_{2}\left(k_{0}\right)$, caractérisée par [7, (6.4.1)] :

$$
\begin{cases}\operatorname{tr} \overline{\widetilde{\lambda}}(\widetilde{\theta})(x)=(q-1) \widetilde{\theta}(x) & \text { si } x \in k_{0}^{\times} \\ \operatorname{tr} \widetilde{\widetilde{\lambda}}(\widetilde{\theta})(x n)=-\widetilde{\theta}(x) & \text { si } x \in k_{0}^{\times}, n \in N\left(k_{0}\right)-\{\operatorname{id}\} \\ \operatorname{tr} \widetilde{\widetilde{\lambda}}(\widetilde{\theta})(x)=-(\widetilde{\theta}(x)+\widetilde{\theta}(\gamma(x))) & \text { si } x \in \ell^{\times}-k_{0}^{\times} .\end{cases}
$$

Alors $\tilde{\lambda}$ est le relèvement de $\overline{\widetilde{\lambda}}(\widetilde{\theta})$ à $\widetilde{U}_{0}(\mathcal{L})$. Elle est de caractère central trivial sur $\mathfrak{o}_{0}^{\times}$et est $\tau$-invariante :

$$
\forall g \in \widetilde{U}_{0}(\mathcal{L}), \quad \widetilde{\lambda}(\tau(g))=\widetilde{\lambda}\left((\operatorname{dét} g)^{-1} g\right)=\widetilde{\lambda}(g)
$$

Elle possède deux prolongements à $\widetilde{J} \tau$-invariants, $\widetilde{\Lambda}$ et $\widetilde{\Lambda}^{\prime}$, qui diffèrent par leur valeur en $\varpi: \widetilde{\Lambda}(\varpi)=\omega_{\lambda}(-1)=-\widetilde{\Lambda}^{\prime}(\varpi)$.

On note alors

$$
\widetilde{\pi}=\operatorname{Ind}_{\widetilde{J}} \widetilde{G} \widetilde{\Lambda} \quad \text { et } \quad \widetilde{\pi}^{\prime}=\operatorname{Ind}_{\widetilde{J}} \widetilde{G} \widetilde{\Lambda}^{\prime}
$$

On prolonge $\widetilde{\pi}$ et $\widetilde{\pi}^{\prime}$ à $\widetilde{G} \Gamma$ en imposant $\widetilde{\Lambda}(\tau)=\widetilde{\Lambda}^{\prime}(\tau)=\widetilde{\lambda}(\tau)=1$ (4.2.1). En notant $E$ l'extension quadratique non ramifiée de $F$, dont le groupe multiplicatif $E^{\times}$est plongé dans le normalisateur de $\mathfrak{a}_{0}(\mathcal{L})$, on a immédiatement :

$$
\forall x \in E^{\times}, x \notin F^{\times}, \quad \operatorname{tr} \widetilde{\Lambda}(x)=\operatorname{tr} \lambda\left(N_{\tau}(x)\right) .
$$

4.3. Construction dans le cas de niveau strictement positif. C'est l'analogue de [4] dans le cas de dimension deux.

On considère les sous-groupes ouverts compacts modulo le centre de $\widetilde{G}$, $\Gamma$-invariants :

$$
\begin{aligned}
& \widetilde{H}_{1}=\left(1+\mathfrak{p}_{E}\right) \widetilde{U}_{\left[\frac{n}{2}\right]+1}(\mathcal{L}), \quad \widetilde{J}_{1}=\left(1+\mathfrak{p}_{E}\right) \widetilde{U}_{\left[\frac{n+1}{2}\right]}(\mathcal{L}), \\
& \widetilde{J}_{c}=\mathfrak{o}_{E}^{\times} \widetilde{U}_{\left[\frac{n+1}{2}\right]}(\mathcal{L}), \quad \widetilde{J}=E^{\times} \widetilde{U}_{\left[\frac{n+1}{2}\right]}(\mathcal{L})=E^{\times} \widetilde{J}_{c} .
\end{aligned}
$$

Sur $\widetilde{H}_{1}$, on considère le caractère $\widetilde{\theta}=\theta \circ \mathcal{N}_{\tau}$ [4, cor. 3.2]. Si $n$ est pair, il existe une unique représentation $\widetilde{\eta}$ de $\widetilde{J}_{1}$ contenant $\widetilde{\theta}$, nécessairement $\tau$ invariante. Si $n$ est impair, on pose $\widetilde{\eta}=\widetilde{\theta}$.

Puisque la dimension de $\widetilde{\eta}$ et l'ordre de $\widetilde{J}_{c} / \widetilde{J}_{1}$ sont premiers entre eux, il existe des prolongements de $\widetilde{\eta}$, et en particulier des prolongements $\tau$-invariants de caractère central trivial sur $\mathfrak{o}_{0}^{\times}$. Deux tels prolongements ont même restriction au sous-groupe $\widetilde{J}_{0}=\mathfrak{o}_{0}^{\times} N_{E_{\mid L}}\left(\mathfrak{o}_{E}^{\times}\right) \widetilde{J}_{1}$. On distingue alors trois cas :

(nr-nr) $F$ n'est pas ramifiée sur $F_{0}$ : l'extension $E$ est alors ramifiée et $\widetilde{\eta}$ est de dimension 1 . De plus, $\mu$ est d'ordre 2 et les deux changements de base de $\pi$ ont même caractère central égal à $\widetilde{\omega_{\lambda}}$. On impose donc que 
le caractère central de $\widetilde{\Lambda}$ soit $\widetilde{\omega_{\lambda}}$. Il existe alors deux prolongements de $\widetilde{\omega_{\lambda}} \widetilde{\eta}$ à $\widetilde{J}$ : ils ont même restriction $\widetilde{\lambda}$ à $\widetilde{J}_{c}$ et sont égaux à \pm 1 en $\varpi_{L}$.

(r-r) $F$ est ramifiée sur $F_{0}$ et $n$ est impair : l'extension $E$ n'est pas ramifiée sur $F$ et $\varpi^{-n} b$ est un élément de $\mathfrak{o}_{E}^{\times}$invariant par $\sigma$ : $L=F_{0}\left[\varpi^{-n} b\right]$ n'est pas ramifiée sur $F_{0}$ et $E$ est ramifiée sur $L$. Le groupe $\widetilde{J}_{0}$ est d'indice 2 dans $\widetilde{J}_{c}$ et $\widetilde{J}=F^{\times} \widetilde{J}_{c}$. Il existe quatre prolongements $\tau$-invariants de $\widetilde{\eta}=\widetilde{\theta}$ à $\widetilde{J}$ de caractère central trivial sur $F_{0}^{\times}$, deux de caractère central $\widetilde{\omega_{\lambda}}$ et deux de caractère central $(-1)^{\text {val }} \widetilde{\omega_{\lambda}}$. Mais, si l'on considère l'autre prolongement de $\eta$ à $J$, on retrouve ces mêmes prolongements de $\widetilde{\eta}$.

(r-nr) $F$ est ramifiée sur $F_{0}$ et $n$ est pair : l'extension $E$ n'est pas ramifiée sur $F$ et $L$ est engendrée sur $F_{0}$ par $\varpi^{-n+1} b$, une uniformisante de $E$. Donc $E$ n'est pas ramifiée sur $L$. Le groupe $\widetilde{J}_{0}$ est d'indice $q+1$ dans $\widetilde{J}_{c}$ et $\widetilde{J}=F^{\times} \widetilde{J}_{c}$. Il existe $2(q+1)$ prolongements $\tau$-invariants de $\widetilde{\eta}$ à $\widetilde{J}$ de caractère central trivial sur $F_{0}^{\times}$, une moitié de caractère

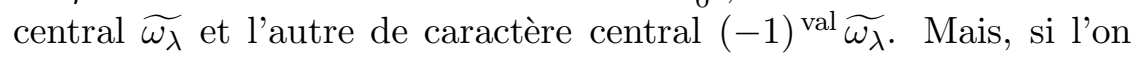
considère les $(q+1)$ prolongements de $\eta$ à $J$ on retrouve les mêmes prolongements de $\widetilde{\eta}$.

Remarque. Ces trois cas, auxquels on ne cesse de faire référence par la suite, se repèrent par la ramification de $F$ sur $F_{0}$ puis celle de $E$ sur $E^{\sigma}$. Les deux premiers cas correspondent à des paires $(E / F, \sigma)$ "paire" au sens de la définition 1.2 de 12 tandis que le dernier cas correspond à une paire $(E / F, \sigma)$ "impaire", tout comme le cas de niveau 0 vu précédemment. Cette différence se reflète dans les énoncés des proposition 4.4 et théorème 4.5 .

Lemme. Il existe une unique représentation $\widetilde{\Lambda}$ de $\widetilde{J} \cdot \Gamma$ vérifiant :

(i) $\widetilde{\Lambda}_{\mid \widetilde{J}}$ est un prolongement $\tau$-invariant de $\widetilde{\eta}$, de caractère central $\widetilde{\omega_{\lambda}}$,

(ii) pour tout $x \in E^{\times}, \operatorname{tr} \widetilde{\Lambda}(x \tau)=\operatorname{tr} \lambda\left(\mathcal{N}_{\tau}(x)\right)$.

On note alors

$$
\widetilde{\pi}=\operatorname{Ind} \widetilde{G}_{\widetilde{J}}^{\widetilde{\Lambda}} \widetilde{J}_{\mid \widetilde{J}}
$$

que l'on prolonge à $\widetilde{G} \Gamma \operatorname{par} \operatorname{Ind} \underset{\widetilde{J} \Gamma}{\widetilde{G} \Gamma} \widetilde{\Lambda}$.

Démonstration. On rappelle [4, lemme 3.3] qu'il existe un unique prolongement de $\widetilde{\eta}$ à $\widetilde{J}_{1} \Gamma$ tel que :

$$
\forall g \in \widetilde{J}_{1}, \operatorname{tr} \widetilde{\eta}(g \tau)=\operatorname{tr} \eta\left(\mathcal{N}_{\tau}(g)\right) .
$$

Soit $\widetilde{\Lambda}$ un prolongement de $\widetilde{\eta}$ de caractère central $\widetilde{\omega_{\lambda}}$. On le prolonge à $\widetilde{J} \cdot \Gamma$ en imposant : $\widetilde{\Lambda}(\tau)=\widetilde{\eta}(\tau)$. Dans les cas (nr-nr) et (r-r), $\widetilde{\Lambda}$ est de dimension 1 et la condition (ii) du lemme équivaut à : $\widetilde{\Lambda}\left(\varpi_{E}\right)=1$ dans le cas (nr-nr) ; $\widetilde{\Lambda}(\zeta)=1$ où $\zeta$ est une racine primitive $\left(q^{2}-1\right)$-ième de 1 contenue dans $E$ dans le cas $(\mathrm{r}-\mathrm{r})$. Ceci définit un et un seul prolongement de la liste.

Dans le cas (r-nr), seule la restriction $\widetilde{\lambda}$ de $\widetilde{\Lambda}$ à $\widetilde{J}_{c}$ importe. Le calcul de la trace de $\lambda$ sur les éléments de $\mathfrak{o}_{E_{\mid L}}^{1}$ est effectué au paragraphe 5 (5.3.4. (2)). 
Il existe donc un unique caractère $\xi$ de $\mathfrak{o}_{E_{\mid L}}^{1} H_{1}$, prolongeant $\theta$ tel que :

$$
\forall x \in \mathfrak{o}_{E_{\mid L}}^{1}, x \notin \mathfrak{o}_{\mid F_{0}}^{1}, \operatorname{tr} \lambda(x)=\epsilon \xi(x),
$$

où $\epsilon$ est égal à -1 si $\operatorname{dim} \eta=q, 1$ sinon.

Pour le calcul de $\operatorname{tr} \widetilde{\lambda}$, on distingue deux cas suivant la dimension de $\widetilde{\lambda}$. Si $\widetilde{\lambda}$ est de dimension $1, \lambda$ l'est également et le résultat est immédiat. Supposons donc $\tilde{\lambda}$ de dimension $q$. On introduit alors le groupe $\mathcal{X}$ des caractères de $\widetilde{J}_{c} / \mathfrak{o}^{\times} \widetilde{J}_{1}$ qui s'identifie au groupe des caractères de $k_{E}^{\times}$triviaux sur $k^{\times}$, groupe cyclique d'ordre $q+1$ engendré par un caractère $\tau$-invariant $\widetilde{\kappa}$. Grâce aux calculs effectués en 5.3.2 avec les notations précisées au (3) de 5.3.4, on est assuré de l'existence de $q+1$ entiers $m_{i}$ de somme $q$ tels que :

$$
\forall x \in \mathfrak{o}_{E}^{\times}, \operatorname{tr} \widetilde{\lambda}(x)=\oplus_{i=0}^{q} m_{i}\left(\widetilde{\kappa}^{i} \widetilde{\xi}\right)(x) .
$$

Chaque composante isotypique étant $\tau$-invariante, il existe $q+1$ entiers $n_{i}$ tels que :

$$
\forall x \in \mathfrak{o}_{E}^{\times}, \operatorname{tr} \widetilde{\lambda}(x \tau)=\oplus_{i=0}^{q} n_{i}\left(\widetilde{\kappa}^{i} \widetilde{\xi}\right)(x) \quad \text { et } \quad \sum_{i=0}^{q} n_{i}=\operatorname{tr} \widetilde{\eta}(\tau)=\operatorname{dim} \eta .
$$

On raisonne alors comme dans la démonstration de la proposition 3.5 de [4], et on obtient :

- si $\operatorname{dim} \eta=1$, tous les entiers $n_{i}$ sont nuls sauf un égal à 1 ;

- si $\operatorname{dim} \eta=q$, tous les entiers $n_{i}$ sont égaux à 1 sauf un qui est nul.

Il existe donc un et un seul prolongement $\widetilde{\lambda}$ de $\widetilde{\eta}$ à $\widetilde{J}_{c}$ tel que :

$$
\forall x \in \mathfrak{o}_{E}^{\times}, x \notin \mathfrak{o}^{\times}, \operatorname{tr} \widetilde{\lambda}(x \tau)=\varepsilon \widetilde{\xi}(x)=\operatorname{tr} \lambda\left(\mathcal{N}_{\tau}(x)\right) .
$$

Ceci termine la démonstration du lemme.

\subsection{Stabilité.}

Proposition. Soit $\widetilde{\pi}=\operatorname{Ind}_{\widetilde{J}} \widetilde{G} \widetilde{\Lambda}$ la représentation de $G L_{2}(F)$ définie en (4.2.2) ou (4.3.1).

(i) Elle appartient à l'image du changement de base stable si et seulement si

- elle est de niveau 0 ou

- elle est de niveau strictement positif et l'extension E associée est non ramifiée sur $F$ et sur $L:=E^{\sigma}$.

(ii) Lorsque $\widetilde{\pi}$ appartient à l'image du changement de base labile, $\widetilde{\pi} \cdot \mu^{-1}$ odét appartient à l'image du changement de base stable.

Démonstration. L'assertion (ii) n'est qu'une redite de [18, §11.4]. Pour l'assertion (i), on distingue deux cas.

On suppose d'abord que $\widetilde{\Lambda}$ est de dimension 1 . Alors par [7, §19] $\widetilde{\pi}$ est associée à la paire admissible $\left(E, \widetilde{\Lambda}_{\mid E^{\times}}\right)$. Mais $E$ est soit d'indice de ramification 2 sur $F$, soit non ramifiée sur $F$ mais ramifiée sur $L$. Autrement dit la paire $(E / F, \sigma)$ est paire au sens de [12, déf. 1.2]. Comme $\widetilde{\Lambda}$ est triviale sur $L^{\times}$(lemme 4.3 (ii)), $\widetilde{\pi}$ est $G L_{2}\left(F_{0}\right)$-distinguée [12, Théorème 1.1] donc $\widetilde{\pi}$ appartient au changement de base labile [10, Théorème 7].

On suppose maintenant que $\widetilde{\Lambda}$ est de dimension au moins 2 . Il n'y a plus de rapport simple entre la trace et la trace tordue de $\widetilde{\Lambda}$ ce qui rend l'emploi 
du critère précédent moins adapté que celui de la définition. Grâce à [18, il n'y a que deux possibilités : soit le caractère tordu de $\widetilde{\pi}$, soit celui de $\widetilde{\pi} \cdot \mu \circ$ dét $^{-1}$ est constant sur les classes de $\tau$-conjugaison stable de $\widetilde{G}$. On peut donc déduire si $\widetilde{\pi}$ appartient à l'image du changement de base stable en évaluant son caractère tordu en deux éléments $g$ et $g^{\prime}$ stablement $\tau$-conjugués et tels que $\operatorname{tr} \widetilde{\pi}(g \tau) \neq 0$ et $\mu \circ$ dét $\left(g^{-1} g^{\prime}\right) \neq 1$.

On choisit $g=\zeta\left(\zeta\right.$ est une racine primitive $\left(q^{2}-1\right)$-ième de 1 contenue dans $\left.\mathfrak{o}_{E}^{\times}\right)$et $g^{\prime}=\varpi_{L} g$ où $\varpi_{L}=\zeta^{\frac{q+1}{2}} \varpi$. Tous deux sont de norme cyclique $x=\zeta^{1-q}$ et $\mu \circ \operatorname{dét}\left(\varpi_{L}\right)=\mu \circ N_{L_{\mid F_{0}}}\left(\varpi_{L}\right)=-1$. Notons que $x \in \widetilde{U}_{0}(\mathcal{L})$ est très régulier : $\quad \forall h \in \widetilde{G}, \quad h^{-1} x h \in \widetilde{U}_{0}(\mathcal{L}) \Rightarrow h \in F^{\times} \widetilde{U}_{0}(\mathcal{L})$.

Par conséquent, si $\widetilde{\pi}$ est de niveau 0, la formule de Mackey donne :

$$
\operatorname{tr} \widetilde{\pi}(\zeta \tau)=\sum_{\substack{h \in \widetilde{G} / \widetilde{J} \\ h^{-1} \zeta \tau(h) \in \widetilde{J}}} \operatorname{tr} \widetilde{\Lambda}\left(h^{-1} \zeta \tau(h) \tau\right)=\operatorname{tr} \widetilde{\Lambda}(\zeta \tau)=\operatorname{tr} \lambda(x)
$$

et de même, $\operatorname{tr} \widetilde{\pi}\left(\zeta \varpi_{L} \tau\right)=\omega_{\lambda}(-1) \operatorname{tr} \widetilde{\pi}\left(\zeta^{\frac{q+3}{2}} \tau\right)=\omega_{\lambda}(-1) \operatorname{tr} \lambda(-x)=\operatorname{tr} \widetilde{\pi}(\zeta \tau)$.

D'après la définition de $\lambda$ (cf. $4.1(\mathrm{a})$ ), $\operatorname{tr} \lambda(x)$ n'est pas nul dès que le caractère $\theta$ associé n'est pas d'ordre 4 . Si $\theta$ est d'ordre $4(q \equiv-1 \bmod 4)$, on refait le même raisonnement avec $g=\zeta^{2}$ sachant que $\operatorname{tr} \widetilde{\pi}\left(\zeta^{2} \tau\right) \neq 0$.

Quand $\tilde{\pi}$ est de niveau 0, elle appartient à l'image du changement de base stable.

Supposons maintenant que $\widetilde{\pi}$ est de niveau strictement positif. Notons $\widetilde{K}(\mathcal{L})$ le normalisateur dans $\widetilde{G}$ de l'ordre associé à $\mathcal{L}, \widetilde{K}(\mathcal{L})=F^{\times} \widetilde{U}_{0}(\mathcal{L})$, et

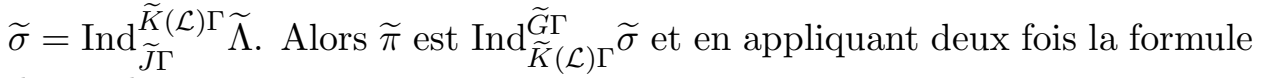
de Mackey, on a :

$$
\begin{gathered}
\operatorname{tr} \widetilde{\pi}(\zeta \tau)=\operatorname{tr} \widetilde{\sigma}(\zeta \tau)=\sum_{\substack{h \in \widetilde{U}_{0}(\mathcal{L}) / \widetilde{J}_{c} \\
h^{-1} \zeta \tau(h) \in \widetilde{J}_{c}}} \operatorname{tr} \widetilde{\Lambda}\left(h^{-1} \zeta \tau(h) \tau\right) \\
\text { et } \operatorname{tr} \widetilde{\pi}\left(\zeta \varpi_{L} \tau\right)=\omega_{\lambda}(-1) \sum_{\substack{h \in \widetilde{U}_{0}(\mathcal{L}) / \widetilde{J}_{c} \\
h^{-1} \zeta^{\frac{q+3}{2}} \tau(h) \in \widetilde{J}_{c}}} \operatorname{tr} \widetilde{\Lambda}\left(h^{-1} \zeta^{\frac{q+3}{2}} \tau(h) \tau\right)
\end{gathered}
$$

On reprend alors le raisonnement de [4, §4.7] en remarquant que $\mathcal{G} a l\left(E / F_{0}\right)$ est abélien (car d'ordre 4). On obtient :

$$
\begin{aligned}
\operatorname{tr} \tilde{\pi}(\zeta \tau) & =\sum_{\gamma \in \mathcal{G} a l(E / F)} \operatorname{tr} \tilde{\lambda}(\gamma(\zeta) \tau)=\operatorname{tr} \lambda(x)+\operatorname{tr} \lambda\left(x^{-1}\right) \\
& =\omega_{\lambda}(-1)\left(\operatorname{tr} \lambda(-x)+\operatorname{tr} \lambda\left(-x^{-1}\right)\right)=\operatorname{tr} \widetilde{\pi}\left(\zeta \varpi_{L} \tau\right)
\end{aligned}
$$

Or $\lambda$ est associé à un caractère $\xi$ par (4.3.2) : ou bien $\xi(x)+\xi\left(x^{-1}\right) \neq 0$, ou bien $\xi\left(x^{2}\right)+\xi\left(x^{-2}\right) \neq 0$. On procède comme dans le cas de niveau 0 .

\subsection{Description des changements de base.}

Théorème. Soit $(\pi, \mathcal{V})$ une représentation irréductible très cuspidale de $G$ et $(J, \lambda)$ un type simple maximal définissant $\pi$ comme au paragraphe ${ }_{4.1}$, (a) ou (b). On note $\widetilde{\pi}=\operatorname{Ind}_{\widetilde{J}} \widetilde{\Lambda} \widetilde{\Lambda}$ la représentation irréductible cuspidale de 
$\widetilde{G}$ définie en (4.2.2) ou (4.3.1) selon que $\pi$ est de niveau 0 ou non.

(i) $F$ n'est pas ramifiée sur $F_{0}$ : l'image de $\pi$ par le changement de base stable est $\tilde{\pi} \cdot \mu^{-1}$ odét tandis que celle par le changement de base labile est $\widetilde{\pi}$.

(ii) $F$ est ramifiée sur $F_{0}$ et $E$ est ramifiée sur $E^{\sigma}$ : on note $\chi$ le caractère d'ordre 2 de $J$ trivial sur $J_{1}$ et on pose : $\pi^{\prime}=\operatorname{Ind}_{J}^{G} \lambda \cdot \chi$ et $\widetilde{\pi}^{\prime}=\operatorname{Ind}_{\widetilde{J}} \widetilde{G} \widetilde{\Lambda} \cdot \widetilde{\chi}$. L'image de $\pi$ par le changement de base stable est $\tilde{\pi} \cdot \mu^{-1} \circ$ dét si $q \equiv 1$ $\bmod 4, \widetilde{\pi}^{\prime} \cdot \mu^{-1}$ o dét sinon. L'image de $\widetilde{\pi}$ par le changement de base labile est alors $\widetilde{\pi}$ si $q \equiv 1 \bmod 4$ et $\widetilde{\pi}^{\prime}$ sinon.

(iii) $F$ est ramifiée sur $F_{0}$ et $E$ n'est pas ramifiée sur $E^{\sigma}$ (y compris cas de niveau 0) : l'image de $\pi$ par le changement de base stable est $\tilde{\pi}$, celle par le changement de base labile $\tilde{\pi} \cdot \mu \circ$ dét.

Suit la démonstration de ce théorème.

4.6. Grâce à [18, §11.4] il suffit d'établir le résultat pour le changement de base stable. Notons $\widetilde{\pi}_{s t}$ la représentation de $\widetilde{G}$ préssentie être l'image de $\pi$ par le changement de base stable dans le théorème et montrons qu'elle vérifie bien l'identité de caractères décrivant ce dernier [18, $\S \S 4.11,12.5$ et 11.4], c'est-à-dire : pour tout $g \in \widetilde{G}$ dont la norme cyclique $\mathcal{N}_{\tau}(g)$ est régulière elliptique et tout $x \in \mathcal{N}_{\tau}(g)$,

$$
\operatorname{tr} \widetilde{\pi}_{s t}(g \tau)=c_{\tau}\left(\widetilde{\pi}_{s t}\right) \operatorname{tr} \pi(x),
$$

où $\widetilde{\pi}_{s t}(\tau)$ est un opérateur d'entrelacement d'ordre 2 entre $\widetilde{\pi}_{s t}$ et $\widetilde{\pi}_{s t}^{\tau}$ et $c_{\tau}\left(\widetilde{\pi}_{s t}\right)$ un signe ne dépendant que du choix de $\widetilde{\pi}_{s t}(\tau)$. Dans la construction précédente, on a choisi $\widetilde{\pi}_{s t}(\tau)$ pour que $c_{\tau}\left(\widetilde{\pi}_{s t}\right)$ soit égal à 1 .

Soit $g \in \widetilde{G}$. On suppose que $x=N_{\tau}(g)$ est un élément de $G$, régulier et elliptique. On note $T\left(F_{0}\right)$ le centralisateur de $x$ dans $G$. C'est un tore compact de $G$ et $T(F)$ est $\tau$-invariant et isomorphe soit au groupe multiplicatif d'une extension quadratique $E_{x}$ de $F$, soit à $F^{\times} \times F^{\times}$.

La formule de Mackey fournit une expression des traces de $\pi(x)$ et $\widetilde{\pi}(g \tau)$ : (4.6.2)

$$
\operatorname{tr} \pi(x)=\sum_{\substack{y \in G / J \\ y^{-1} x y \in J}} \operatorname{tr} \lambda\left(y^{-1} x y\right) \quad \text { et } \quad \operatorname{tr} \widetilde{\pi}_{s t}(g \tau)=\sum_{\substack{h \in \widetilde{G} / \widetilde{J} \\ h^{-1} g \tau(h) \in \widetilde{J}}} \operatorname{tr} \widetilde{\Lambda}_{s t}\left(h^{-1} g \tau(h) \tau\right) .
$$

4.7. On suppose d'abord que $\pi$ est de niveau strictement positif. La démonstration est semblable à celle du théorème 3.7 de [4].

On introduit le sous-groupe distingué de $\widetilde{G}$, noté $\widetilde{G}^{+}$, défini comme le noyau du caractère $\mu \circ$ dét. Pour tout sous-groupe $H$ de $\widetilde{G}$, on abrège $H \cap \widetilde{G}^{+}$en $H^{+}$.

Lemme. Soient $g \in \widetilde{G}$ et $x \in G$ comme ci-dessus. On note $\mathcal{C} l_{\tau}^{s t}(g)$ la classe de $\tau$-conjugaison stable de $g$ et $\mathcal{C} l^{s t}(x)$ la classe de conjugaison stable de $x$.

(i) Le groupe $\widetilde{G}^{+}$est réunion de classes de $\tau$-conjugaison.

(ii) Si $\mu$ o dét est d'ordre $2, \widetilde{G}^{+}$rencontre la classe de $\tau$-conjugaison stable de $g$ et $\mathcal{C l}_{\tau}^{s t}(g)^{+}$contient la moitié des classes de $\tau$-conjugaison contenues dans $\mathcal{C} l_{\tau}^{s t}(g)$.

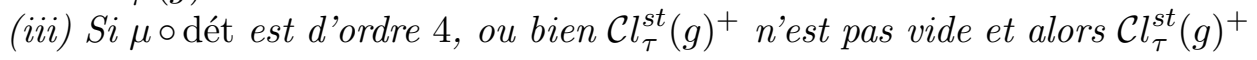


contient la moitié des classes de $\tau$-conjugaison contenues dans $\mathcal{C} l_{\tau}^{s t}(g)$; ou bien $\mathcal{C} l_{\tau}^{\text {st }}(g)$ ne rencontre pas $\widetilde{G}^{+}$, et alors :

$$
\mathcal{C} l_{\tau}^{s t}(g) \cap \widetilde{J}=\emptyset \quad \text { et } \quad \mathcal{C} l^{s t}(x) \cap J=\emptyset .
$$

(iv) Chaque classe de $\tau$-conjugaison dans $\mathcal{C} l_{\tau}^{\text {st }}(g)^{+}$contient deux classes de $\tau$ - $\widetilde{G}^{+}$-conjugaison.

Démonstration. Pour la première assertion, il suffit de remarquer que $\mu \circ$ dét est constant sur les classes de $\tau$-conjugaison.

Pour la suite, on sait que les classes de $\tau$-conjugaison de $g$ dans $\mathcal{C} l_{\tau}^{s t}(g)$ sont paramétrées par $H^{1}(\Gamma, T(F))$ : à un cocycle $c$, on associe la classe de $\tau$ conjugaison de $c(\tau) g$.

Si $T(F) \simeq E_{x}^{\times}$, il y a deux classes de $\tau$-conjugaison, celle de $g$ et celle de $a g$ où $a \in L_{x}^{\times}, a \notin N_{E_{x \mid L_{x}}}\left(E_{x}^{\times}\right)$et $L_{x}=E_{x}^{\sigma}$. Notons que $L_{x}$ est une extension quadratique de $F_{0}$, nécessairement distincte de $F$. Mais alors, dét $a$ appartient à $N_{L_{x \mid F_{0}}}\left(L_{x}^{\times}\right)$sans être un carré de $F_{0}$ donc $\mu \circ$ dét( $(a)$ est égal à -1 . Ainsi, $\mu \circ$ dét prend deux valeurs opposées sur les deux classes de $\tau$-conjugaison.

Si $T(F) \simeq F^{\times} \times F^{\times}$, il y a quatre classes de $\tau$-conjugaison, celles de $g$, $\left(1, \alpha_{0}\right) g,\left(\alpha_{0}, 1\right) g$ et $\left(\alpha_{0}, \alpha_{0}\right) g$. Encore une fois $\mu \circ$ dét prend deux valeurs opposées.

Par conséquent, si $\mu$ odét est d'ordre 2, la moitié des classes de $\tau$-conjugaison dans $\mathcal{C} l_{\tau}^{s t}(g)$ sont contenues dans $\widetilde{G}^{+}$.

Supposons que $\mu \circ$ dét est d'ordre 4 . Ou bien $\mu \circ$ dét $(g) \in\{ \pm 1\}$ et la moitié des classes de $\tau$-conjugaison dans $\mathcal{C} l_{\tau}^{s t}(g)$ sont contenues dans $\widetilde{G}^{+}$; ou bien $\mu \circ \operatorname{dét}(g) \in\{ \pm i\}$ et $\mathcal{C} l_{\tau}^{s t}(g)^{+}$est vide. On termine la démonstration en remarquant que : $\mu \circ \operatorname{dét}(\widetilde{J})=\{ \pm 1\}$ et $\operatorname{dét}(J) \subset 1+\mathfrak{p}$. En effet, l'hypothèse sur dét $g$ implique que celui-ci est de valuation impaire et par suite, que dét $x$ appartient à $-1+\mathfrak{p}$.

(iv) Si $g_{1}$ et $g_{2}$ sont deux éléments de $\widetilde{G}^{+} \tau$ - $\widetilde{G}$-conjugués par deux éléments $h_{1}$ et $h_{2}$ alors $h_{1} h_{2}^{-1}$ appartient au $\tau$-centralisateur de $g_{1}$, en particulier la norme de son déterminant est 1 . Par conséquent, si $\mu$ est d'ordre $2, h_{1}$ et $h_{2}$ diffèrent d'un élément de $\widetilde{G}^{+}$. Si $\mu$ est d'ordre 4 , le $\tau$-centralisateur de $g_{1}$ contient un élément $a$ tel que $\mu \circ \operatorname{dét}(a)=-1$ (cf. (ii) et (iii)) et $h_{1}$ et $h_{2}$ diffèrent d'un élément de $\widetilde{G}^{+}$ou de $\widetilde{G}^{+} a$. Dans les deux cas, il y a deux classes de $\tau$ - $\widetilde{G}^{+}$-conjugaison dans une classe de $\tau$-conjugaison.

Dans le cas où $\mathcal{C} l_{\tau}^{s t}(g)^{+}$est vide, l'égalité (4.6.1) est satisfaite puisque les deux sommes de (4.6.2) sont nulles. On peut donc supposer que $\mathcal{C} l_{\tau}^{s t}(g)^{+}$ n'est pas vide et, quitte à changer $g$ dans sa classe de $\tau$-conjugaison stable sans changer de $x$, que $g \in \widetilde{G}^{+}$.

Notons $\mathcal{C}_{J}^{s t}(x)$ l'ensemble des classes de $J$-conjugaison contenues dans $\mathcal{C} l^{s t}(x) \cap J$ et $\mathcal{C}_{\tau, \widetilde{J}^{+}}^{s t}(g)$ celui des classes de $\tau$ - $\widetilde{J}^{+}$-conjugaison contenues dans $\mathcal{C} l_{\tau}^{s t}(g) \cap \widetilde{J}^{+}$. Notons également $n(x)$ (resp. $\widetilde{n}(g)$ ) le nombre de classes de conjugaison (resp. $\tau$-conjugaison) contenues dans $\mathcal{C} l^{s t}(x)\left(\right.$ resp. $\left.\mathcal{C} l_{\tau}^{s t}(g)\right)$. En 
suivant le raisonnement de [4, §4.2], on a :

$$
\begin{aligned}
\operatorname{tr} \pi(x)=\frac{1}{n(x)} \sum_{x^{\prime} \in \mathcal{C}_{J}^{s t}(x)} c\left(x^{\prime}\right) \operatorname{tr} \lambda\left(x^{\prime}\right) \\
\text { et } \operatorname{tr} \widetilde{\pi}_{s t}(g \tau)=\frac{1}{\widetilde{n}(g)} \sum_{g^{\prime} \in \mathcal{C}_{\tau, \widetilde{J}^{+}}^{\text {st }}(g)} \widetilde{c}\left(g^{\prime}\right) \operatorname{tr} \widetilde{\Lambda}_{s t}\left(g^{\prime} \tau\right)
\end{aligned}
$$

où $\quad c\left(x^{\prime}\right)=\left[T\left(F_{0}\right): T\left(F_{0}\right) \cap y J y^{-1}\right]$ si $x^{\prime}=y^{-1} x y, y \in G$

et $\quad \widetilde{c}\left(g^{\prime}\right)=\left[T\left(F_{0}\right): T\left(F_{0}\right) \cap h \widetilde{J} h^{-1}\right]$ si $g^{\prime}=h^{-1} g \tau(h), h \in \widetilde{G}$.

4.8. On introduit les sous-groupes $J^{\prime}$ et $\widetilde{J}^{\prime}$ de $J$ et $\widetilde{J}^{+}$respectivement, en distinguant deux cas :

cas 1: $F / F_{0}$ est non ramifiée ou $q \equiv 1 \bmod 4$. Dans ce cas, $J^{\prime}=F_{\mid F_{0}}^{1} J_{1}$ et $\widetilde{J}^{\prime}=F^{\times} \widetilde{J}_{1}$

cas $2: F / F_{0}$ est ramifiée et $q \equiv-1 \bmod 4$. Alors $J^{\prime}=J_{1}$ et $\widetilde{J}^{\prime}=F_{0}^{\times} \widetilde{J}_{1}$.

Dans les cas où $J$ est plus grand que $J^{\prime}$ ou $\widetilde{J}^{+}$plus grand que $N_{E_{\mid L}}\left(\mathfrak{o}_{E}^{\times}\right) \widetilde{J}^{\prime}$, c'est-à-dire lorsqu'on se trouve dans le cas (r-nr) ou le cas (r-r) et $q \equiv-1$ mod 4, on considére les représentations par "paquets" fabriqués de la façon suivante.

Du côté de $G$, on considère tous les prolongements à $J$ de la restriction à $J^{\prime}$ de $\lambda$. Ils sont au nombre de $d=\left[J: J^{\prime}\right]$ et de la forme $\lambda \otimes \kappa^{r}, 0 \leq r \leq d-1$, où $\kappa$ est un caractère de $J$, trivial sur $J^{\prime}$ et d'ordre $d$ (qui s'identifie à un caractère du groupe engendré par $\zeta^{q-1}$ dans le cas (r-nr), -1 dans le cas $(\mathrm{r}-\mathrm{r}))$.

Notons $\widetilde{\kappa}$ le caractère de $\widetilde{J}$ que l'on obtient sur $\widetilde{J}$ en relevant le caractère $\kappa \circ N_{\tau}$ de $\widetilde{J} / N_{E_{\mid L}}\left(\mathfrak{o}_{E}^{\times}\right) \widetilde{J}^{\prime}$. On remplace $\pi$ et $\widetilde{\pi}_{s t}$ par :

$$
\begin{aligned}
& \pi=\oplus_{r=0}^{d-1} \pi_{r} \quad \text { où } \quad \pi_{r}=\operatorname{Ind}_{J}^{G} \lambda \otimes \kappa^{r} \\
& \widetilde{\pi}_{s t}=\oplus_{r=0}^{d-1} \widetilde{\pi}_{r} \quad \text { où } \quad \widetilde{\pi}_{r}=\operatorname{Ind}_{\widetilde{J}} \widetilde{G} \widetilde{\Lambda}_{s t} \otimes \widetilde{\kappa}^{r}
\end{aligned}
$$

Alors, en sommant les expressions (4.7.1) des caractères des $\pi_{r}$ d'une part, et des $\widetilde{\pi}_{r}$ d'autre part, on obtient :

$$
\begin{aligned}
& \operatorname{tr} \pi(x)=\frac{1}{n(x)} \sum_{x^{\prime} \in \mathcal{C}_{J}^{s t}(x) \cap J^{\prime}} d c\left(x^{\prime}\right) \operatorname{tr} \lambda\left(x^{\prime}\right)=\frac{1}{n(x)} \sum_{x^{\prime} \in \mathcal{C}_{J^{\prime}}^{s t}(x)} \frac{d}{d\left(x^{\prime}\right)} c\left(x^{\prime}\right) \operatorname{tr} \lambda\left(x^{\prime}\right) \\
& \text { et } \operatorname{tr} \widetilde{\pi}_{s t}(g \tau)=\frac{1}{\widetilde{n}(g)} \sum_{g^{\prime} \in \mathcal{C}_{\tau, \widetilde{J}^{+}}^{s t}(g) \cap N_{E_{\mid L}}\left(\mathfrak{o}_{E}^{\times}\right) \widetilde{J}^{\prime}} d \widetilde{c}\left(g^{\prime}\right) \operatorname{tr} \widetilde{\Lambda}_{s t}\left(g^{\prime} \tau\right) \\
& =\frac{1}{\widetilde{n}(g)} \sum_{\substack{g^{\prime} \in \mathcal{C}_{\tau, N_{E}}^{s t} \\
E_{\mid L}\left(\mathfrak{o}_{E}^{\times}\right) \widetilde{J}^{\prime}}} \frac{d}{\widetilde{d}\left(g^{\prime}\right)} \widetilde{c}\left(g^{\prime}\right) \operatorname{tr} \widetilde{\Lambda}_{s t}\left(g^{\prime} \tau\right)
\end{aligned}
$$

où $d\left(x^{\prime}\right)$ est le nombre de classes de $J^{\prime}$-conjugaison dans l'intersection de la classe de $J$-conjugaison de $x^{\prime}$ avec $J^{\prime}$ et $\widetilde{d}\left(g^{\prime}\right)$ celui des classes de $\tau$ $N_{E_{\mid L}}\left(\mathfrak{o}_{E}^{\times}\right) \widetilde{J}^{\prime}$-conjugaison dans l'intersection de la classe de $\tau-\widetilde{J}^{+}$-conjugaison de $g^{\prime}$ avec $N_{E_{\mid L}}\left(\mathfrak{o}_{E}^{\times}\right) \widetilde{J}^{\prime}$. 
Remarquons que les nombres $d\left(x^{\prime}\right)$ et $\widetilde{d}\left(g^{\prime}\right)$ sont invariants quand on conjugue $x^{\prime}$ par un élément de $J$, respectivement $\tau$-conjugue $g^{\prime}$ par un élément de $N_{E_{\mid L}}\left(\mathfrak{o}_{E}^{\times}\right) \widetilde{J}^{\prime}$.

On uniformise les notations en posant $\widetilde{d}\left(g^{\prime}\right)=d\left(x^{\prime}\right)=1$ dans les cas non mentionnés dans ce paragraphe.

4.9. L'étape suivante consiste à remplacer $N_{E_{\mid L}}\left(\mathfrak{o}_{E}^{\times}\right) \widetilde{J}^{\prime}$ par $\widetilde{J^{\prime}}$. Elle ne concerne que le cas (r-r).

Lemme. Dans le cas ( $r-r)$,

$$
\sum_{g^{\prime} \in \mathcal{C}_{\tau, N_{E} s \mid}^{s t}\left(\mathfrak{o}_{E}^{\times}\right) \widetilde{J}^{\prime}} \frac{d}{\widetilde{d}\left(g^{\prime}\right)} \widetilde{c}\left(g^{\prime}\right) \operatorname{tr} \widetilde{\Lambda}_{s t}\left(g^{\prime} \tau\right)=\sum_{g^{\prime} \in \mathcal{C}_{\tau, \widetilde{J}^{\prime}}^{s t}(g)} \frac{d}{\widetilde{d}\left(g^{\prime}\right)} \widetilde{c}\left(g^{\prime}\right) \operatorname{tr} \widetilde{\Lambda}_{s t}\left(g^{\prime} \tau\right) .
$$

Démonstration. Considérons l'application $\Psi \operatorname{de} \mathcal{C}_{\tau, \widetilde{J}^{\prime}}^{s t}(g) \operatorname{dans} \mathcal{C}_{\tau, N_{E_{\mid L}}}^{s t}\left(\mathfrak{o}_{E}^{\times}\right) \widetilde{J}^{\prime}(g)$ qui à la classe de $\tau$ - $\widetilde{J}^{\prime}$-conjugaison de $g^{\prime} \in \mathcal{C} l_{\tau}^{s t}(g) \cap \widetilde{J}^{\prime}$ associe la classe de $\tau$ - $N_{E_{\mid L}}\left(\mathfrak{o}_{E}^{\times}\right) \widetilde{J}^{\prime}$-conjugaison de $g^{\prime}$. Etudions d'abord son image.

Soit $g^{\prime} \in N_{E_{\mid L}}\left(\mathfrak{o}_{E}^{\times}\right) \widetilde{J}^{\prime}$. L'ensemble des classes à droite modulo $\widetilde{J}^{\prime}$ dans $N_{E_{\mid L}}\left(\mathfrak{o}_{E}^{\times}\right) \widetilde{J}^{\prime}$ est représenté par $\left\{\zeta^{2 r}, r \in \llbracket 0, \frac{q-1}{2} \rrbracket\right\}$. Il existe donc $r \in \llbracket 0, \frac{q-1}{2} \rrbracket$ et $h \in \widetilde{J}^{\prime}$ tels que : $g^{\prime}=\zeta^{2 r} h=\zeta^{r}\left(\zeta^{r} h \zeta^{-r}\right) \tau\left(\zeta^{-r}\right)$. L'élément $\zeta^{r} h \zeta^{-r}$ appartient à $\widetilde{J}^{\prime}$ tandis que $\zeta^{r}$ appartient à $N_{E_{\mid L}}\left(\mathfrak{o}_{E}^{\times}\right) \widetilde{J}^{\prime}$ si et seulement si $r$ est pair. Donc si $r$ est pair, $g^{\prime}$ appartient à $\operatorname{Im} \Psi$. Réciproquement, les classes de $\tau$ - $\widetilde{J}^{\prime}$-conjugaison dans la classe de $\tau-N_{E_{\mid L}}\left(\mathfrak{o}_{E}^{\times}\right) \widetilde{J}^{\prime}$-conjugaison de $g^{\prime}$ ont un représentant de la forme $\zeta^{-2 s} g^{\prime} \tau\left(\zeta^{2 s}\right)=\zeta^{2 r-4 s}\left(\zeta^{2 s} h \zeta^{-2 s}\right)$ pour un $s \in \llbracket 0, \frac{q-1}{2} \rrbracket$. L'une d'entre elles est contenue dans $\widetilde{J}^{\prime}$ si et seulement il existe $s \in \llbracket 0, \frac{q-1}{2} \rrbracket$ tel que $\zeta^{2 r-4 s} \in F_{0}^{\times}$, c'est-à-dire $r-2 s \equiv 0 \bmod \left(\frac{q+1}{2}\right)$.

Si $q \equiv 1 \bmod 4, g^{\prime}$ est donc $\tau-N_{E_{\mid L}}\left(\mathfrak{o}_{E}^{\times}\right) \widetilde{J}^{\prime}$-conjugué à un élément de $\widetilde{J}^{\prime}: \Psi$ est surjective.

Si $q \equiv-1 \bmod 4, g^{\prime}$ est $\tau$ - $N_{E_{\mid L}}\left(\mathfrak{o}_{E}^{\times}\right) \widetilde{J}^{\prime}$-conjugué à un élément de $\widetilde{J^{\prime}}$ si et seulement si $r$ est pair.

Etudions les fibres de $\Psi$. Soient $g_{1}, g_{2}$ deux éléments de $\widetilde{J}^{\prime}$ qui sont $\tau$ $N_{E_{\mid L}}\left(\mathfrak{o}_{E}^{\times}\right) \widetilde{J}^{\prime}$-conjugués. Quitte à $\tau$ - $\widetilde{J}^{\prime}$-conjugué $g_{1}$, on peut supposer qu'il existe $r \in \llbracket 0, \frac{q-1}{2} \rrbracket$ tel que $\zeta^{2 r} \tau$-conjugue $g_{2}$ en $g_{1}$. Mais alors $\zeta^{-4 r}$ appartient à $\widetilde{J}^{\prime}$, c'est-à-dire $\frac{q+1}{2}$ divise $2 r$.

Si $q \equiv 1 \bmod 4, \frac{q+1}{2}$ divise $r$ et $g_{1}=g_{2}: \Psi$ est injective. En remarquant que $\Psi$ conserve la valeur de $\frac{d}{\widetilde{d}\left(g^{\prime}\right)} \widetilde{c}\left(g^{\prime}\right) \operatorname{tr} \widetilde{\Lambda}_{s t}\left(g^{\prime} \tau\right)$, on établit l'égalité (4.9.1).

Si $q \equiv-1 \bmod 4, \frac{q+1}{4}$ divise $r$ et $g_{2}$ est égal à $g_{1}$ ou $\zeta^{-\frac{q+1}{2}} g_{1} \tau\left(\zeta^{\frac{q+1}{2}}\right):$ les fibres de $\Psi$ sont de cardinal 2 .

Dans ce cas, on remarque que l'application $g^{\prime} \mapsto \zeta^{-1} g^{\prime} \tau(\zeta)$ définit une bijection de $\operatorname{Im} \Psi$ sur son complémentaire dans $\mathcal{C}_{\tau, N_{E_{\mid L}}\left(\mathfrak{o}_{E}^{\times}\right) \widetilde{J}^{\prime}}^{s t}(g)$ qui préserve la valeur de $\frac{d}{d\left(g^{\prime}\right)} \widetilde{c}\left(g^{\prime}\right) \operatorname{tr} \widetilde{\Lambda}_{s t}\left(g^{\prime} \tau\right)$. On retrouve donc l'égalité (4.9.1).

4.10. Il reste à étudier la restriction de l'application $N_{\tau}$ à $\mathcal{C}_{\tau, \widetilde{J}^{\prime}}^{s t}(g)$. 
Lemme. Soient $g$ et $x$ comme précédemment.

(i) Soit $g^{\prime} \in \widetilde{J}^{\prime}$. Il existe $h \in \widetilde{J^{\prime}}$ tel que $N_{\tau}\left(h^{-1} g^{\prime} \tau(h)\right) \in J^{\prime}$.

(ii) Soient $x_{1}, x_{2}$ deux éléments de $J^{\prime}$ conjugués sous $\widetilde{J}^{\prime}$. Alors ils sont conjugués sous $J^{\prime}$.

(iii) Soit $x^{\prime} \in \mathcal{C} l^{s t}(x) \cap J^{\prime}$. Il existe $g^{\prime} \in \mathcal{C} l_{\tau}^{s t}(g) \cap \widetilde{J}^{\prime}$ tel que $: N_{\tau}\left(g^{\prime}\right)=x^{\prime}$. Il en existe deux à $\tau$ - $\widetilde{J}^{\prime}$-conjugaison près dans le cas 1 et 4 dans le cas 2.

Démonstration. On se place dans le cas 1.

(i) On a $g^{\prime}=z j$ où $z \in F^{\times}, j \in \widetilde{J}_{1}$. Par le lemme 3.1 de [4], il existe $h \in \widetilde{J}_{1}$ tel que $N_{\tau}\left(h^{-1} j \tau(h)\right) \in J_{1}$. Alors $N_{\tau}\left(h^{-1} g^{\prime} \tau(h)\right) \in J^{\prime}$.

(ii) Notons pour $i=1$ ou $2, x_{i}=u_{i} j_{i}$ où $u_{i} \in F_{\mid F_{0}}^{1}, j_{i} \in J_{1}$. Soit $h \in \widetilde{J}^{\prime}$ tel que $x_{2}=h^{-1} x_{1} h$. On peut prendre $h \in \widetilde{J}_{1}$. Alors :

$x_{2}=h^{-1} x_{1} h \Leftrightarrow u_{1}^{-1} u_{2}=h^{-1} j_{1} h j_{2}^{-1} \in F_{\mid F_{0}}^{1} \cap \widetilde{J}_{1} \Leftrightarrow u_{1}=u_{2}$ et $j_{2}=h^{-1} j_{1} h$.

Par le lemme 3.1(ii) déjà cité, on peut choisir $h \in J_{1}$.

(iii) Notons $x^{\prime}=u j$ avec $u \in F_{\mid F_{0}}^{1}$ et $j \in J_{1}$. Par le théorème de Hilbert 90 et encore le lemme 3.1(ii), il existe $g^{\prime}=z \widetilde{j}, z \in F^{\times}$et $\widetilde{j} \in \widetilde{J}_{1}$ tel que : $N_{\tau}\left(g^{\prime}\right)=x^{\prime}$. De plus, $g^{\prime} \in \mathcal{C} l_{\tau}^{s t}(g)$.

Soient maintenant $g_{1}^{\prime}, g_{2}^{\prime} \in \mathcal{C} l_{\tau}^{s t}(g) \cap \widetilde{J}^{\prime}$ tels que : $N_{\tau}\left(g_{1}\right)=N_{\tau}\left(g_{2}\right)=x^{\prime}$. Alors $g_{i}, i=1,2$, s'écrit $z_{i} j_{i}$ où $z_{i} \in F^{\times}$et $j_{i} \in \widetilde{J}_{1}$ tel que $j_{i} \tau\left(j_{i}\right) \in J_{1}$. L'égalité précédente donne alors :

$$
\frac{z_{1} \bar{z}_{2}}{\bar{z}_{1} z_{2}}=j_{2} \tau\left(j_{2}\right)\left(j_{1} \tau\left(j_{1}\right)\right)^{-1} \Leftrightarrow z_{1} \bar{z}_{2} \in F_{0}^{\times} \text {et } j_{1} \tau\left(j_{1}\right)=j_{2} \tau\left(j_{2}\right)
$$

quitte à multiplier $z_{1}$ et $j_{1}$ par un élément de $1+\mathfrak{p}$ sans changer leur produit. Encore une fois, $j_{1}$ et $j_{2}$ sont donc $\tau$ - $\widetilde{J}_{1}$-conjugués. Alors $g_{1}$ et $g_{2}$ sont $\tau$ - $\widetilde{J}^{\prime}$-conjugués si et seulement si $z_{1} \bar{z}_{2}$ est une norme de $F^{\times}$dans $F_{0}$. A $\tau$ - $\widetilde{J}^{\prime}$-conjugaison près, on a deux $g^{\prime} \in \widetilde{J}^{\prime}$ de norme cyclique $x^{\prime}$.

On se place dans le cas 2. Les assertions (i) et (ii) sont immédiates. Soit $x^{\prime} \in \mathcal{C} l^{s t}(x) \cap J^{\prime}$. Alors il existe $j \in \widetilde{J}_{1}$, unique à $\tau$ - $\widetilde{J}_{1}$-conjugaison près, tel que $N_{\tau}(j)=x^{\prime}$. Mais pour tout $u \in F_{0}^{\times}, u j$ est de norme cyclique $x^{\prime}$ et $u j$ est $\tau$ - $\widetilde{J}^{\prime}$-conjugué à $j$ si et seulement si $u$ est un carré dans $F_{0}^{\times}$.

En effet, si $u$ est un carré, il est clair que $j$ et $u j$ sont $\tau$ - $\widetilde{J}^{\prime}$-conjugués. Réciproquement, on peut supposer que $u$ est $1, \varpi_{0}, \zeta^{q+1}$ ou $\varpi_{0} \zeta^{q+1}$. Si $j$ et $u j$ sont $\tau-\widetilde{J}^{\prime}$-conjugués, il existe $v \in F_{0}^{\times}$et $j^{\prime} \in \widetilde{J}_{1}$ tels que $u j=$ $v^{-1}\left(j^{\prime-1} j \tau\left(j^{\prime}\right)\right) \tau(v)$. Mais alors, $u v^{2} \in \widetilde{J}_{1} \cap F_{0}^{\times}=1+\mathfrak{p}_{0}$ donc $u=1$.

4.11. Etablissons l'égalité (4.6.1). $\mathrm{Si} \mathcal{C} l_{\tau}^{s t}(g) \cap \widetilde{J}^{\prime}$ est vide, $\mathcal{C} l^{s t}(x)$ ne rencontre pas $J^{\prime}$ (lemme4.10, (i)) donc les deux sommes de (4.7.1) sont nulles. Supposons maintenant que $g \in \widetilde{J}^{\prime}$ et $x \in J^{\prime}$ (lemme 4.10, (i)). Alors l'application $\mathcal{N}_{\tau}$ induit une surjection de $\mathcal{C}_{\tau, \widetilde{J}^{\prime}}^{s t}(g)$ sur $\mathcal{C}_{J^{\prime}}^{s t}(x)$ dont les fibres sont de cardinal 2 dans le cas 1, 4 dans le cas 2 (lemme 4.10). De plus, tr $\widetilde{\Lambda}$ est constant sur ces fibres et l'identité s'obtient comme dans [4, $\S 4.5$ et 4.6 ] en ajoutant le résultat suivant :

Lemme. Soit $g^{\prime} \in \widetilde{J}^{\prime}$ tel que $x^{\prime}:=N_{\tau}\left(g^{\prime}\right) \in J^{\prime}$. Alors $\widetilde{d}\left(g^{\prime}\right)$ est égal à $d\left(x^{\prime}\right)$ dans le cas 1, $2 d\left(x^{\prime}\right)$ dans le cas 2. 
Démonstration. Le résultat est immédiat dans les cas (nr-nr) et (r-r) quand $q \equiv 1 \bmod 4$.

Dans le cas (r-r) quand $q \equiv-1 \bmod 4, J$ est le produit $\{ \pm 1\} J^{\prime}$ donc $d\left(x^{\prime}\right)$ vaut 1 . D'autre part, $N_{E_{\mid L}}\left(\mathfrak{o}_{E}^{\times}\right) \widetilde{J}^{\prime}$ est un sous-groupe d'indice 2 de $\widetilde{J}^{+}$donc la classe de $\tau$ - $\widetilde{J}^{+}$-conjugaison de $g^{\prime}$ contient au plus deux classes de $\tau$ - $N_{E_{\mid L}}\left(\mathfrak{o}_{E}^{\times}\right) \widetilde{J}^{\prime}$-conjugaison, à savoir celles de $g^{\prime}$ et de $-\zeta^{-1} g^{\prime} \tau(\zeta)$. Remarquons qu'il existe un entier $r$ tel que $g^{\prime} \in \zeta^{(q+1) r} \varpi_{0}^{\mathbb{Z}} \widetilde{J}_{1}$ et que toute la classe de $\tau$ - $N_{E_{\mid L}}\left(\mathfrak{o}_{E}^{\times}\right) \widetilde{J}^{\prime}$-conjugaison de $g^{\prime}$ est contenue dans $\bigcup_{s \equiv 0[4]} \zeta^{s} \varpi_{0}^{\mathbb{Z}} \widetilde{J}_{1}$. Mais, $-\zeta^{-1} g^{\prime} \tau(\zeta)$ appartient à $\zeta^{s} \varpi_{0}^{\mathbb{Z}} \widetilde{J}_{1}$ où $s=\frac{q^{2}-1}{2}-2+(q+1) r \equiv 2 \bmod 4$. Par suite : $\widetilde{d}\left(g^{\prime}\right)=2=2 d\left(x^{\prime}\right)$.

Dans le cas (r-nr), $g^{\prime}$ s'écrit $z g_{1}^{\prime}$ avec $z \in\left(F^{\times}\right)^{+}$et $g_{1}^{\prime} \in \widetilde{J}_{1}$. Alors $x^{\prime}=\frac{z}{\bar{z}} x_{1}^{\prime}$ où $x_{1}^{\prime}=N_{\tau}\left(g_{1}^{\prime}\right) \in J_{1}$. Puisque $z$ et $\frac{z}{\bar{z}}$ sont centraux dans $\widetilde{J}^{+}$et $J$ respectivement, on a : $\widetilde{d}\left(g^{\prime}\right)=\widetilde{d}\left(g_{1}^{\prime}\right)$ et $d\left(x^{\prime}\right)=d\left(x_{1}^{\prime}\right)$. On peut donc supposer que $g^{\prime} \in \widetilde{J}_{1}$ et $x^{\prime} \in J_{1}$. Sous cette hypothèse, $\widetilde{J}^{\prime}$ contient toute la classe de $\tau-\widetilde{J}^{+}$-conjugaison de $g^{\prime}$. D'après le lemme 4.10, $N_{\tau}$ induit une application de l'ensemble des classes de $\tau$ - $\widetilde{J}^{\prime}$-conjugaison contenues dans $\mathcal{C} l_{\tau-\widetilde{J}^{+}}\left(g^{\prime}\right)$ dans l'ensemble des classes de $J^{\prime}$-conjugaison contenues dans $\mathcal{C} l_{J}\left(x^{\prime}\right)$. Cette application est clairement surjective car tout $x^{\prime \prime} \in \mathcal{C} l_{J}\left(x^{\prime}\right)$ s'écrit $j^{-1} x^{\prime} j$, $j \in J$, et est l'image par $N_{\tau}$ de $g^{\prime \prime}:=j^{-1} g^{\prime} \tau(j)$, élément appartenant à $\mathcal{C} l_{\tau-\widetilde{J}^{+}}\left(g^{\prime}\right) \cap \widetilde{J}_{1}$.

Etudions la fibre en $x^{\prime \prime}$. D'après le lemme 4.10, elle contient au plus 2 classes si $q \equiv 1 \bmod 4,4$ si $q \equiv-1 \bmod 4$ et, d'après la démonstration de ce même lemme, ces classes de $\tau$ - $\widetilde{J}^{\prime}$-conjugaison sont représentées par : $g^{\prime \prime}$ et $\alpha_{0} g^{\prime \prime}$ si $q \equiv 1 \bmod 4 ; u g^{\prime \prime}$ où $u$ parcourt un ensemble de représentants des classes de $F_{0}^{\times}$modulo ses carrés. Mais, une de ces classes est contenue dans $\mathcal{C} l_{\tau-\widetilde{J}^{+}}\left(g^{\prime}\right)$ si et seulement si son représentant $z g^{\prime \prime}$ est $\tau-\widetilde{J}^{+}$-conjugué à $g^{\prime \prime}$, c'est-à-dire il existe $h=(\varpi \zeta)^{r} \zeta^{2 s} y, r, s \in \mathbb{Z}$ et $y \in \widetilde{J}_{1}$ tel que :

$$
\begin{gathered}
z g^{\prime \prime}=\left((\varpi \zeta)^{r} \zeta^{2 s} y\right)^{-1} g^{\prime \prime} \tau\left((\varpi \zeta)^{r} \zeta^{2 s} y\right) \Leftrightarrow z=\varpi_{0}^{-r} \zeta^{-(q+1)(r+2 s)} \quad \bmod \widetilde{J}_{1} \\
\Leftrightarrow z=\varpi_{0}^{-r} \zeta^{-(q+1)(r+2 s)} \bmod 1+\mathfrak{p}_{0} \Leftrightarrow\left\{\begin{array}{l}
r=0 \text { et } z=1 \quad \text { si } q \equiv 1 \bmod 4 \\
z=1 \text { ou } z=\varpi_{0} \zeta^{q+1} \text { si } q \equiv-1 \bmod 4
\end{array}\right.
\end{gathered}
$$

Ainsi, la fibre en $x^{\prime \prime}$ est de cardinal 1 si $q \equiv 1 \bmod 4,2$ sinon.

A ce point, sont démontrées les assertions du théorème 4.5 correspondant aux cas (nr-nr) et (r-r) lorsque $q$ est congru à 1 modulo 4 .

4.12. Dans les autres cas de niveau strictement positif, chaque représentation $\widetilde{\pi}_{r}$ est l'image par le changement de base stable d'une représentation $\pi_{r}^{\prime \prime}$ [18, \$11.4.1] caractérisée par l'identité (4.6.1). D'après ce qui précède, pour tout $x \in G$ régulier, elliptique, de la forme $N_{\tau}(g)$ pour un $g \in \widetilde{G}$,

$$
\sum_{i=0}^{d-1} \operatorname{tr} \pi_{i}^{\prime \prime}(x)=\sum_{i=0}^{d-1} \operatorname{tr} \pi_{i}(x) .
$$


Alors, comme au paragraphe 4.7 de [4, on obtient que les images des représentations $\pi_{r}, 0 \leq r \leq d-1$, par le changement de base stable sont les représentations $\widetilde{\pi}_{r}, 0 \leq r \leq d-1$.

Dans le cas (r-r) où $q$ est congru à -1 modulo 4 , les deux représentations $\widetilde{\pi}_{0}$ et $\widetilde{\pi}_{1}$ ne sont autres que $\widetilde{\pi} \cdot \mu^{-1}$ o dét et $\widetilde{\pi}^{\prime} \cdot \mu^{-1}$ o dét et seule $\widetilde{\pi}^{\prime} \cdot \mu^{-1} \circ$ dét a pour caractère central $\widetilde{\omega}_{\pi}$. Elle est donc l'image de $\pi$ par le changement de base stable.

Dans le cas (r-nr), lorsque $q$ est congru à -1 modulo 4, l'argument du caractère central permet d'affirmer que l'image de $\pi$ par le changement de base stable appartient à $\left\{\widetilde{\pi}_{r}, r \equiv 0 \bmod 2\right\}$. Sans hypothèse sur $q$, pour montrer que l'image de $\pi$ est bien $\widetilde{\pi}_{0}$, il suffit d'évaluer leurs caractères en des éléments bien choisis. Comme dans la démonstration de la proposition 4.4, on choisit $g=\zeta$ ou $\zeta^{2}$. Alors, par (4.4.1) et en poursuivant le raisonnement comme en [4, §4.7] :

$$
\operatorname{tr} \widetilde{\pi}(g \tau)=\operatorname{tr} \lambda(x)+\operatorname{tr} \lambda\left(x^{-1}\right)=\operatorname{tr} \pi(x) \neq 0 .
$$

Ceci termine la démonstration du théorème dans le cas des représentations de niveau strictement positif.

4.13. Considérons le cas où $\pi$ est de niveau 0 . La représentation $\widetilde{\pi}$ est une représentation $\tau$-invariante, à caractère central trivial sur $F_{0}$ et de caractère stable : elle appartient donc à l'image du changement de base stable et son antécédent est nécessairement une représentation de niveau 0 décrite par 4.1 (a), conséquence de tout ce qui précède. Ces dernières se distinguent par la restriction de leurs caractères en les éléments de $G$ dont le groupe des points sur $F$ du centralisateur est le groupe multiplicatif d'une extension quadratique de $F$, éléments dits "elliptiques" dans [11] et "très elliptiques" ici pour éviter les confusions.

Soit $g \in \widetilde{G}$ tel que $x=g \tau(g)$ est un élément de $G$ régulier et très elliptique. Le groupe $T(F)$ des points sur $F$ du centralisateur de $x$ est le groupe multiplicatif d'une extension quadratique $E$ de $F$, nécessairement non ramifiée sur $F$.

Si $\mathcal{C} l_{\tau}^{s t}(g) \cap \widetilde{J}$ est vide, alors $\mathcal{C} l^{s t}(x) \cap J$ aussi. En effet, un élément $x^{\prime}$ de $\mathcal{C} l^{s t}(x) \cap J$ est de la forme $N_{\tau}\left(g^{\prime}\right)$ où $g^{\prime} \in \mathcal{C} l_{\tau}^{s t}(g)$ et commute à $x^{\prime}$ (puisque $g$ commute à $x)$. Or $x^{\prime}$ est très elliptique donc $g^{\prime}$ appartient à $F^{\times} \widetilde{U}_{0}(\mathcal{L})=\widetilde{J}$ [11, th. 1]. Pour un tel $g$, l'identité (4.6.1) est satisfaite.

Si $\mathcal{C} l_{\tau}^{s t}(g) \cap \widetilde{J}$ n'est pas vide, on considère $g^{\prime} \in \mathcal{C} l_{\tau}^{s t}(g) \cap \widetilde{J}$ et $x^{\prime}=N_{\tau}\left(g^{\prime}\right)$. Alors $x^{\prime} \in \widetilde{U}_{0}(\mathcal{L})$ et $x^{\prime}$ est $\widetilde{G}$-conjugué à $x: x^{\prime}=y^{-1} x y, y \in \widetilde{G}$.

Notons que $\tau\left(x^{\prime}\right)$ appartient à $\widetilde{U}_{0}(\mathcal{L})$ et $\tau\left(x^{\prime}\right)=\left(y^{-1} \tau(y)\right)^{-1} x^{\prime} y^{-1} \tau(y)$. Ainsi $y^{-1} \tau(y) \in F^{\times} \widetilde{U}_{0}(\mathcal{L})$, autrement dit : $\tau(y)=y j, j \in \widetilde{J}$. Mais alors la chaîne de réseaux $y \mathcal{L}$ est une chaîne autoduale de $F^{2}$ et $x \in U_{0}(y \mathcal{L})$. De deux choses l'une :

- la chaîne $y \mathcal{L}$ est de même invariant que $\mathcal{L}$ auquel cas il existe $y_{0} \in G$ tel que $y \mathcal{L}=y_{0} \mathcal{L}: y_{0}^{-1} x y_{0} \in U_{0}(\mathcal{L})=J$

- la chaîne $y \mathcal{L}$ n'est pas de même invariant que $\mathcal{L}$ auquel cas il existe $y_{0} \in G$ tel que $y \mathcal{L}=y_{0} \mathcal{L}^{\prime}$ où $\mathcal{L}^{\prime}$ est une chaîne de période 1 telle que $U_{0}\left(\mathcal{L}^{\prime}\right)$ contient le même sous-groupe d'Iwahori $\mathcal{I}$ que $U_{0}(\mathcal{L}): y_{0}^{-1} x y_{0} \in U_{0}\left(\mathcal{L}^{\prime}\right)$. Mais, $\mathcal{L}^{\prime}$ est d'invariant pair et $U_{0}\left(\mathcal{L}^{\prime}\right)$ est la réunion disjointe de $\mathcal{I}$ et de son 
complémentaire, le premier formé des éléments de déterminant dans $1+\mathfrak{p}$, le second formé des éléments de déterminant dans $-1+\mathfrak{p}$. Or le déterminant de $y_{0}^{-1} x y_{0}$ est égal à dét $g^{\prime} / \overline{\operatorname{dét} g^{\prime}}$ où dét $g^{\prime}$ est nécessairement de valuation paire, donc est un élément de $1+\mathfrak{p}$. Ainsi, $y_{0}^{-1} x y_{0}$ appartient à $\mathcal{I} \subset U_{0}(\mathcal{L})$. Dans les deux cas, $\mathcal{C} l^{s t}(x) \cap J$ n'est pas vide. On peut donc choisir $x \in J$ puis $g \in \widetilde{J}$. Par la formule de Mackey et puisque $x$ est très elliptique, on obtient : $\quad \operatorname{tr} \widetilde{\pi}(g \tau)=\operatorname{tr} \widetilde{\Lambda}(g \tau) \quad$ et $\operatorname{tr} \pi(x)=\operatorname{tr} \lambda(x)$.

On conclut grâce à (4.2.3).

\section{Caractères de Représentations irréduCtibles de CERTAines EXTENSIONS DE GROUPES FINIS.}

On présente dans ce paragraphe un calcul de caractères de représentations admissibles de groupes compacts qui permet de justifier ou de se convaincre de la validité d'affirmations contenues dans le paragraphe 3.1 (cas (b')) et la démonstration du lemme 4.3 .

Le calcul exposé est plus complexe que nécessaire ici. Mais, on rencontre cette même question dans l'étude du transfert de $U(1,1)\left(F_{0}\right) \times U(1)\left(F_{0}\right)$ à $U(2,1)\left(F_{0}\right)$. On a donc choisi un cadre suffisament grand pour réutiliser les résultats lors de cette étude.

5.1. Données, hypothèses et objectif. On fixe un nombre premier $p$.

Soient $\mathbb{J}$ un groupe fini, $\mathbb{J}_{1}$ un sous-groupe distingué de $\mathbb{J}$ de centre $Z$ et $\mathbb{J}^{\prime}$ un sous-groupe de $\mathbb{J}$ tels que $\mathbb{J}=\mathbb{J}^{\prime} \mathbb{J}_{1}$ et $\mathbb{J}^{\prime} \cap \mathbb{J}_{1} \subset Z$. On suppose que :

(i) $\mathbb{J}_{1}$ est un $p$-groupe, extra-spécial de classe 2 ou abélien ;

(ii) $\mathbb{J}^{\prime}=T \mathbb{J}_{1}^{\prime}$ où $T$ un sous-goupe abélien de $\mathbb{J}^{\prime}$ et $\mathbb{J}_{1}^{\prime}$ un $p$-sous-groupe distingué, ou bien extra-spécial de classe 2 dont le centre $Z^{\prime}$ contient $\mathbb{J}_{1}^{\prime} \cap T$, ou bien abélien (dans ce cas on le note aussi $Z^{\prime}$ ) ;

(iii) $\left[\mathbb{J}_{1}^{\prime}, \mathbb{J}_{1}\right]=1$;

(iv) $\mathbb{J}^{\prime} / \mathbb{J}_{1}^{\prime}$ est d'ordre premier à $p$;

(v) $Z^{\prime} Z$ est contenu dans le centre de $\mathbb{J}$ et $\mathbb{J}_{1}^{\prime} \mathbb{J}_{1}$ est un $p$-groupe extraspécial de classe 2 , de centre $Z^{\prime} Z$.

Soient $\theta$ un caractère fidèle de $Z$ et $\theta^{\prime}$ un caractère fidèle de $Z^{\prime}$ tels que $\theta_{\mid Z \cap Z^{\prime}}=\theta_{\mid Z \cap Z^{\prime}}^{\prime}$.

Ainsi, le groupe $Z$ est un groupe cyclique d'ordre $p$ et le quotient $V=\mathbb{J}_{1} / Z$ est un $\mathbb{F}_{p}$-espace vectoriel de dimension paire, muni d'une forme bilinéaire alternée non dégénérée $\langle.,$.$\rangle définie par :$

$$
<., .>: \begin{array}{ccc}
V \times V & \rightarrow & \mu_{p} \\
\left(g Z, g^{\prime} Z\right) & \mapsto & \theta\left(\left[g, g^{\prime}\right]\right) .
\end{array}
$$

Ceci est également valable pour $Z^{\prime}$ "en primant" $V, \mathbb{J}_{1}, Z$ et $\theta$.

On note $\Theta$ le caractère $\theta^{\prime} \cdot \theta$ de $Z^{\prime} Z$ et $\eta_{\Theta}$ la représentation de Heisenberg de $\mathbb{J}_{1}^{\prime} \mathbb{J}_{1}$ de caractère central $\Theta$. Elle est isomorphe au produit tensoriel des représentations de Heisenberg de $\mathbb{J}_{1}$ et $\mathbb{J}_{1}^{\prime}$ de caractères centraux $\theta$ et $\theta^{\prime}$ respectivement. On note $\eta_{\theta}$ et $\eta_{\theta^{\prime}}$ ces deux représentations, $p^{a}$ et $p^{b}$ leurs dimensions respectives.

On suppose que les représentations $\eta_{\Theta}$ et $\eta_{\theta^{\prime}}$ se prolongent en des représentations de $\mathbb{J}$ et $\mathbb{J}^{\prime}$ respectivement. 
Notre but est de définir une bijection entre les prolongements de $\eta_{\Theta}$ à $\mathbb{J}$ et ceux de $\eta_{\theta^{\prime}}$ à $\mathbb{J}^{\prime}$ caractérisée par une identité de caractères.

Dans les deux cas, les prolongements se distinguent par les valeurs de leurs caractères sur les éléments de $T$. On cherche donc à calculer la trace des prolongements de $\eta_{\Theta}$ en un élément de $T$ en fonction de celle des prolongements de $\eta_{\theta^{\prime}}$, au moins dans les situations qui nous sont utiles.

\subsection{Cinq conséquences des données et hypothèses.}

5.2.1. Tout d'abord, de l'hypothèse (iv), on déduit que :

$$
\mathbb{J}^{\prime} \cap \mathbb{J}_{1} \subset \mathbb{J}_{1}^{\prime} \quad \text { d'où } \quad \mathbb{J} / \mathbb{J}_{1}^{\prime} \mathbb{J}_{1} \simeq \mathbb{J}^{\prime} / \mathbb{J}_{1}^{\prime} \simeq T / T \cap Z^{\prime} \quad \text { et } \quad \mathbb{J}^{\prime} \cap \mathbb{J}_{1} \subset Z^{\prime},
$$

le dernier isomorphisme et l'inclusion sont alors conséquences de (ii) et (i) respectivement.

5.2.2. A $x \in \mathbb{J}^{\prime}$, on associe l'automorphisme symplectique de $V$, encore noté $x$, défini par la conjugaison par $x$, le sous-espace $V^{x}$ de $V$ formé des points fixes sous $x$ et le caractère $\chi_{x}$ de $V^{x}$ défini par :

$$
\chi_{x}(v)=\theta([x, v]) \text { pour tout } v \in V^{x} .
$$

Remarquons d'une part, que $\mathbb{J}_{1}^{\prime}$ centralisant $\mathbb{J}_{1}$ (iii), $V^{x}$ et $\chi_{x}$ ne dépendent que de la classe de $x$ modulo $\mathbb{J}_{1}^{\prime}$; d'autre part, que si $x$ n'appartient pas à $\mathbb{J}_{1}^{\prime}$, son ordre $r$ dans $\mathbb{J}^{\prime} / \mathbb{J}_{1}^{\prime}$ est premier à $p$ (par (iv)) et $\chi_{x}$ est trivial. Pour cette dernière assertion, il suffit de remarquer que :

$$
\begin{aligned}
& \text { - pour tout } v \in V^{x}, \text { tout } s \in \mathbb{N}, \chi_{x}(v)^{s}=1 \Leftrightarrow s \equiv 0[p] \text { ou } \chi_{x}(v)=1 \text {; } \\
& \text { - pour tout } v \in V^{x}, \chi_{x}(v)^{r}=\chi_{x^{r}}(v)=1 .
\end{aligned}
$$

Par conséquent, puisque $\theta$ est fidèle, la projection naturelle du sous-groupe $\mathbb{J}_{1}^{x}$ des éléments de $\mathbb{J}_{1}$ invariants par conjugaison par $x$ sur $V^{x}$ est surjective. En primant les notations, on obtient le résultat analogue pour tout élément de T. Ainsi,

$$
\forall x \in \mathbb{J}^{\prime}, \quad\left(\mathbb{J}_{1} / Z\right)^{x} \simeq \mathbb{J}_{1}^{x} / Z \quad \text { et } \quad \forall t \in T, \quad\left(\mathbb{J}_{1}^{\prime} / Z^{\prime}\right)^{t} \simeq \mathbb{J}_{1}^{\prime t} / Z^{\prime}
$$

Il s'en suit que :

$$
\forall t \in T, \quad\left(\mathbb{J}_{1}^{\prime} \mathbb{J}_{1} / Z^{\prime} Z\right)^{t} \simeq \mathbb{J}_{1}^{\prime t} \mathbb{J}_{1}^{t} / Z^{\prime} Z .
$$

5.2.3. Soit $t \in T$. L'application de $V$ dans $V$ qui à $v \in V$ associe $\left[t^{-1}, v\right]$ est linéaire de noyau $V^{t}$. Son image $\mathcal{I}_{t}=\left\{\left[t^{-1}, v\right], v \in V\right\}$ est l'orthogonal de $V^{t}$. De plus, l'action de $T$ par conjugaison sur $\mathbb{J}_{1}$ fournit une représentation de $T$ dans $G L(V)$ triviale sur $T \cap \mathbb{J}_{1}^{\prime}$. Puisque $T / T \cap \mathbb{J}_{1}^{\prime}$ est fini d'ordre premier à $p$ (iv), cette représentation est semi-simple donc somme de caractères. On en déduit que les sous-espaces $V^{t}$ et $\mathcal{I}_{t}$ sont supplémentaires. Par conséquent, pour tout $t \in T$ (et par suite pour tout $x \in \mathbb{J}^{\prime}$ ), la restriction de $\langle., .>$ au sous-espace $V^{t}$ est non dégénérée et la dimension de $V^{t}$ est paire, notée $2 a_{t}$.

5.2.4. Les paires $(\mathbb{J}, \lambda)$ et $\left(\mathbb{J}^{\prime}, \lambda^{\prime}\right)$ sont deux cas de la situation A.1.7 de $[6]$. En utilisant le corollaire A.1.8 et la surjectivité des projections de $\left(\mathbb{J}_{1}^{\prime} \mathbb{J}_{1}\right)^{t}$ sur $\left(\mathbb{J}_{1}^{\prime} \mathbb{J}_{1} / Z^{\prime} Z\right)^{t}$ et de $\mathbb{J}_{1}^{\prime t}$ sur $\left(\mathbb{J}_{1}^{\prime} / Z^{\prime}\right)^{t}$ pour tout $t \in T$ (comme dans la démonstration de la proposition 13.1 de [5]), on obtient :

Lemme. Soit $t \in T$. 
a) $\operatorname{tr} \lambda(t) \neq 0, \quad \operatorname{tr} \lambda^{\prime}(t) \neq 0 \quad$ et $\quad\|\operatorname{tr} \lambda(t)\|=\left|V^{t}\right|^{\frac{1}{2}} \cdot\left|V^{\prime t}\right|^{\frac{1}{2}}$, $\left\|\operatorname{tr} \lambda^{\prime}(t)\right\|=\left|V^{\prime t}\right|^{\frac{1}{2}}$.

b) Soit $y \in \mathbb{J}_{1}^{\prime} \mathbb{J}_{1}$. Alors $\operatorname{tr} \lambda(t y)$ est non nul si et seulement si ty est $\mathbb{J}_{1}^{\prime} \mathbb{J}_{1}$-conjugué à un élément de $t Z^{\prime} Z$.

c) Soit $y^{\prime} \in \mathbb{J}_{1}^{\prime}$. Alors tr $\lambda^{\prime}\left(t y^{\prime}\right)$ est non nul si et seulement si ty $y^{\prime}$ st $\mathbb{J}_{1}^{\prime}$-conjugué à un élément de $t Z^{\prime}$.

5.2.5. Enonçons une dernière conséquence.

Lemme. Deux éléments de $\mathbb{J}^{\prime}$ sont $\mathbb{J}$-conjugués si et seulement s'ils sont $\mathbb{J}^{\prime}$ - conjugués.

Démonstration. Soient $x, x^{\prime}$ deux éléments de $\mathbb{J}^{\prime}$ qui sont $\mathbb{J}$-conjugués. Quitte à conjuguer l'un d'entre eux par un élément de $\mathbb{J}^{\prime}$, on peut supposer que $x$ et $x^{\prime}$ sont $\mathbb{J}_{1}$-conjugués. On écrit $: x=t y$ où $t \in T$ et $y \in \mathbb{J}_{1}^{\prime}$ et $x^{\prime}=j x j^{-1}$ avec $j \in \mathbb{J}_{1}$. Montrons que $x^{\prime}$ et $x$ sont égaux.

Si $x \in \mathbb{J}_{1}^{\prime}$, il suffit d'utiliser (iii). Si $x \notin \mathbb{J}_{1}^{\prime}$, on a grâce à (iii) :

$$
x^{\prime}=t y \cdot y^{-1}\left[t^{-1}, j\right] y \cdot\left[y^{-1}, j\right]=x \cdot\left[t^{-1}, j\right] \text {. }
$$

Ainsi, $\left[t^{-1}, j\right]=x^{-1} x^{\prime}$ appartient à $Z$ ( $\mathbb{J}_{1} \cap \mathbb{J}^{\prime} \subset Z$ par hypothèse), c'està-dire que $j \in V^{t}=V^{x}$. Et puisque $\chi_{x}$ est trivial (\$5.2.2) et $\theta$ fidèle, on conclut que $x$ et $x^{\prime}$ sont égaux.

Par les lemmes 5.2.4 et 5.2.5, on obtient :

Corollaire. Soit $x$ un élément de $\mathbb{J}^{\prime}$. Les quatre propositions suivantes sont équivalentes :

(i) $\operatorname{tr} \lambda(x) \neq 0$;

(ii) $\operatorname{tr} \lambda^{\prime}(x) \neq 0$;

(iii) $x$ est $\mathbb{J}^{\prime}$-conjugué à un élément de $T Z^{\prime}$;

(iv) $x$ est $\mathbb{J}$-conjugué à un élément de $T Z^{\prime}$.

\subsection{Comparaison de traces.}

5.3.1. Soit $\lambda$ un prolongement de $\eta_{\Theta}$ à $\mathbb{J}$. Notons $\mathbb{J}_{0}^{\prime}$ le centralisateur de $\mathbb{J}_{1}$ dans $\mathbb{J}^{\prime}$. La restriction de $\lambda$ à $\mathbb{J}_{0}^{\prime}$ est de la forme $\lambda_{0}^{\prime} \cdot \eta_{\theta}$ où $\lambda_{0}^{\prime}$ est un prolongement de $\eta_{\theta^{\prime}}$ à $\mathbb{J}_{0}^{\prime}$. Soit $\lambda^{\prime}$ un prolongement de $\lambda_{0}^{\prime}$ à $\mathbb{J}^{\prime}$.

Par la suite, on note $\mathcal{X}$ l'ensemble des caractères de $\mathbb{J}$ triviaux sur $\mathbb{J}_{0}^{\prime} \mathbb{J}_{1}$. Il s'identifie via la restriction à l'ensemble des caractères de $\mathbb{J}^{\prime}$ triviaux sur $\mathbb{J}_{0}^{\prime}$ ou à ceux de $T$ triviaux sur $T \cap \mathbb{J}_{0}^{\prime}$. Selon le contexte, on regarde $\mathcal{X}$ d'une façon ou d'une autre et on note $d$ son cardinal.

Remarquons que lorsque $a=0, \lambda$ est isomorphe à $\lambda^{\prime} \otimes \theta$ pour un prolongement $\lambda^{\prime}$ de $\eta_{\theta^{\prime}}$ convenable. Il existe donc une bijection que l'on peut définir "canoniquement" par l'égalité des traces sur les éléments de $T$.

Si $d=1$, J n'est autre que le produit $\mathbb{J}_{0}^{\prime} \mathbb{J}_{1}$ et $\mathbb{J}^{\prime}$ est égal à $\mathbb{J}_{0}^{\prime}$. La représentation $\lambda$ est de la forme $\lambda^{\prime} \otimes \eta_{\theta}$ pour un unique prolongement $\lambda^{\prime}$ de $\eta_{\theta^{\prime}}$. Il existe donc une bijection que l'on peut définir "canoniquement" par l'identité de caractères suivante :

$$
\forall x \in T, \quad \operatorname{tr} \lambda(x)=p^{a} \operatorname{tr} \lambda^{\prime}(x) .
$$

On suppose donc : $a>0$ et $d>1$. 
5.3.2. On désigne par $\mathbb{K}$ le groupe $\mathbb{J}^{\prime} Z$. Puisque $\lambda$ prolonge $\eta_{\Theta}$, sa restriction à $\mathbb{K}$ est une sous-représentation de la restriction à $\mathbb{K}$ de $\operatorname{Ind}_{\mathbb{J}_{0}^{\prime} \mathbb{J}_{1}}^{\mathbb{J}} \lambda_{0}^{\prime} \otimes \eta_{\theta}$ qui est multiple de $\operatorname{Ind}_{\mathbb{J}_{0}^{\prime}}^{\mathbb{K}} \lambda_{0}^{\prime} \cdot \theta$ d'après la formule de Mackey. Il existe donc $d$ entiers positifs $m_{\xi}(\xi \in \mathcal{X})$ tels que :

$$
\forall k \in \mathbb{K}, \quad \lambda(k)=\bigoplus_{\xi \in \mathcal{X}} m_{\xi}\left(\xi \lambda^{\prime} \cdot \theta\right)(k) \quad \text { et } \quad \sum_{\xi \in \mathcal{X}} m_{\xi}=p^{a}
$$

d'où :

$$
\forall x \in T, \quad \operatorname{tr} \lambda(x)=\operatorname{tr} \lambda^{\prime}(x) \cdot \sum_{\xi \in \mathcal{X}} m_{\xi} \xi(x) .
$$

Notre but est d'évaluer $\sum_{\xi \in \mathcal{X}} m_{\xi} \xi(x)$. De l'assertion $a$ du lemme 5.2.4, on déduit qu'elle est de module $\left|V^{x}\right|^{1 / 2}$ pour tout $x$ de $T$. On obtient alors un système d'équations en les inconnues $m_{\xi}$, à savoir :

$$
\begin{aligned}
& \sum_{\xi \in \mathcal{X}} m_{\xi}=p^{a} \\
& \sum_{\xi \in \mathcal{X}} m_{\xi}^{2}+\sum_{\xi^{\prime} \in \mathcal{X}-\{1\}}\left(\sum_{\xi \in \mathcal{X}} m_{\xi} m_{\xi \xi^{\prime}}\right) \xi^{\prime}(x)=\left|V^{x}\right| \text { pour chaque } \bar{x} \neq 1
\end{aligned}
$$

où $\bar{x}$ représente la classe de $x \in T$ modulo $\mathbb{J}_{0}^{\prime} \cap T$.

5.3.3. On suppose dorénavant que :

(H) $T / \mathbb{J}_{0}^{\prime} \cap T$ est cyclique et que pour tout $\bar{x} \in T / \mathbb{J}_{0}^{\prime} \cap T$ non trivial, $\left|V^{x}\right|=1$.

Alors les $d$ inconnues $M_{1}=\sum_{\xi \in \mathcal{X}} m_{\xi}^{2}-1$ et $M_{\xi^{\prime}}=\sum_{\xi \in \mathcal{X}} m_{\xi} m_{\xi \xi^{\prime}}, \xi^{\prime} \in \mathcal{X}$ non trivial, sont solutions du système linéaire

$$
\left\{\begin{array}{l}
M_{1}+\sum_{\xi^{\prime} \in \mathcal{X}-\{1\}} M_{\xi^{\prime}}=p^{2 a}-1 \\
M_{1}+\sum_{\xi^{\prime} \in \mathcal{X}-\{1\}} \xi^{\prime}(x) M_{\xi^{\prime}}=0
\end{array}\right.
$$

c'est-à-dire : $\forall \xi \in \mathcal{X}, \quad M_{\xi}=\frac{p^{2 a}-1}{d}$. En particulier : $\sum_{\xi \in \mathcal{X}} m_{\xi}^{2}-1=\frac{p^{2 a}-1}{d}$.

Soit $\left\{m_{\xi}, \xi \in \mathcal{X}\right\}$ une solution entière de (5.3.2). Alors :

$$
\sum_{\xi \in \mathcal{X}}\left(\frac{p^{a}+1}{d}-m_{\xi}\right)^{2}=|\mathcal{X}|\left(\frac{p^{a}+1}{d}\right)^{2}-2 \frac{p^{a}+1}{d} \sum_{\xi \in \mathcal{X}} m_{\xi}+\sum_{\xi \in \mathcal{X}} m_{\xi}^{2}=1
$$

i) $\mathrm{Si} \frac{p^{a}+1}{d} \in \mathbb{Z}$, il existe, à permutation près des $m_{\xi}$, deux solutions entières de (5.3.3), à savoir :

$$
m_{1}=\frac{p^{a}+1}{d} \pm 1 \text { et } m_{\xi}=\frac{p^{a}+1}{d} \text { si } \xi \neq 1 ;
$$

et, toujours à permutation près des $m_{\xi}$, il existe une unique solution entière de (5.3.2), à savoir :

$$
m_{1}=\frac{p^{a}+1}{d}-1 \text { et } m_{\xi}=\frac{p^{a}+1}{d} \text { si } \xi \neq 1 .
$$

ii) $\mathrm{Si} \frac{p^{a}+1}{d} \notin \mathbb{Z}$, chaque terme de la somme (5.3.3) appartient à ]0, 1[ donc, pour tout $\xi \in \mathcal{X}, m_{\xi}$ est égal à $u$ ou $u+1$ où $u$ est la partie entière de $\frac{p^{a}+1}{d}$. 
Notons $r$ la différence $\left(p^{a}+1\right)-u d$ et $n$ le nombre d'entiers $m_{\xi}$ égaux à $u$. Remarquons que : $0<r<d$.

De la première équation de (5.3.2), on déduit que $n=d+1-r$, puis de (5.3.3), on obtient :

$$
\begin{aligned}
& \sum_{\xi \in \mathcal{X}}\left(\frac{p^{a}+1}{d}-m_{\xi}\right)^{2}=(d+1-r)\left(\frac{r}{d}\right)^{2}+(r-1)\left(1-\frac{r}{d}\right)^{2}=1 \\
\Leftrightarrow & (r-2)(r-d)=0 \Rightarrow r=2 .
\end{aligned}
$$

Dans ce cas, $d$ divise donc $p^{a}-1$, l'un des $m_{\xi}$ est égal à $\frac{p^{a}-1}{d}+1$ et tous les autres à $\frac{p^{a}-1}{d}$. On obtient ainsi toutes les solutions de (5.3.2).

En conséquence :

Lemme. On suppose l'hypothèse $(H)$ satisfaite. On note $\mathbb{J}_{0}^{\prime}$ le centralisateur de $\mathbb{J}_{1}$ dans $\mathbb{J}^{\prime}$, d le cardinal de $T / \mathbb{J}_{0}^{\prime} \cap T$ et $p^{2 a}$ celui de $\mathbb{J}_{1} / Z$.

Si d divise $p^{a}+1$, il existe un unique prolongement $\lambda^{\prime}$ de $\eta_{\theta^{\prime}}$ tel que:

$$
\forall x \in T, \quad \operatorname{tr} \lambda(x)= \begin{cases}p^{a} \operatorname{tr} \lambda^{\prime}(x) & \text { si } x \in \mathbb{J}_{0}^{\prime} \cap T, \\ -\operatorname{tr} \lambda^{\prime}(x) & \text { sinon. }\end{cases}
$$

Sinon, il existe un unique prolongement $\lambda^{\prime}$ de $\eta_{\theta^{\prime}}$ tel que :

$$
\forall x \in T, \quad \operatorname{tr} \lambda(x)= \begin{cases}p^{a} \operatorname{tr} \lambda^{\prime}(x) & \text { si } x \in \mathbb{J}_{0}^{\prime} \cap T, \\ \operatorname{tr} \lambda^{\prime}(x) & \text { sinon. }\end{cases}
$$

5.3.4. Applications.

(1) L'affirmation de 3.1 (b') demande une justification lorsque la représentation $\lambda$ n'est pas de dimension 1. D'après [3, $\S$ A.6.2], $\lambda$ est de la forme $\lambda_{\theta}$ pour un caractère $\theta$ d'un sous-groupe de $J$. On applique ce qui précède avec :

$$
\mathbb{J}=J / \operatorname{Ker} \theta, \mathbb{J}_{1}=J \cap U_{1}(\mathcal{L}) / \operatorname{Ker} \theta, T=H / \operatorname{Ker} \theta \cap H, \mathbb{J}_{1}^{\prime}=Z^{\prime}=Z .
$$

en remarquant que $T / \mathbb{J}_{0}^{\prime} \cap T \simeq k_{\mid k_{0}}^{1}, V$ est de cardinal $q^{2}$ et le stabilisateur de chaque élément non nul de $V$ est réduit à $\{1\}$.

(2) On reprend les notations de 4.1 (b) et on se place dans le cas (r-nr) du paragraphe 4.3. Pour calculer tr $\lambda$ sur $\mathfrak{o}_{E_{\mid L}}^{1}$, on pose :

$$
\mathbb{J}=J / \operatorname{Ker} \theta, \mathbb{J}_{1}=J_{1} / \operatorname{Ker} \theta, T=\mathfrak{o}_{E_{\mid L}}^{1} / \mathfrak{o}_{E_{\mid L}}^{1} \cap \operatorname{Ker} \theta \text { et } \mathbb{J}_{1}^{\prime}=Z^{\prime}=Z
$$

et on applique le lemme précédent. Remarquons que $\mathbb{J}_{0}^{\prime}$ est alors égal à $\{ \pm 1\} \mathbb{J}_{1}^{\prime}$ donc $d$ vaut $\frac{q+1}{2}$ tandis que $a$ vaut 1 ou 0 suivant que la dimension de $\lambda$ est $q$ ou 1. L'hypothèse (H) est bien satisfaite car $T / T \cap \mathbb{J}_{0}^{\prime}$ est cyclique et tous les éléments de $\mathfrak{o}_{E_{\mid L}}^{1}$ non centraux sont minimaux.

(3) On se place dans la même situation que précédemment mais on s'intéresse cette fois à la représentation $\widetilde{\lambda}$. On suppose de plus que $\widetilde{\lambda}$ n'est pas de dimension 1 (donc $a=1)$. On pose :

$$
\mathbb{J}=\widetilde{J} / \operatorname{Ker} \widetilde{\theta}, \mathbb{J}_{1}=\widetilde{J}_{1} / \operatorname{Ker} \tilde{\theta}, T=\mathfrak{o}_{E}^{\times} / \mathfrak{o}_{E}^{\times} \cap \operatorname{Ker} \tilde{\theta} \text { et } \mathbb{J}_{1}^{\prime}=Z^{\prime}=Z .
$$

C'est un cas particulier de 5.1 et toutes les conséquences qui suivent sont valables, en particulier 5.3.2. 


\section{RÉFÉRENCES}

[1] J. Adler and J. Lansky, Depth-zero base change for unramified $U(2,1)$, J. Number Theory, 114 (2005), 324-360.

[2] J. Adler AND J. LANsky, Depth-zero base change for ramified $U(2,1)$, arXiv:0807.1528v1, 9 Jul 2008.

[3] L. Blasco, Description du dual admissible de $U(2,1)(F)$ par la théorie des types de C. Bushnell et P. Kutzko, Manuscripta Math., 107 (2002), 151-186.

[4] L. Blasco, Types, paquets et changement de base : l'exemple de $U(2,1)\left(F_{0}\right)$, I, Canadian J. Math., 60 (2008), 790-821.

[5] C. Bushnell and G. Henniart, Local tame lifting for GL $(N)$ I: simple characters, Publ. Math. IHES, 83 (1996), 105-233.

[6] C. Bushnell and G. Henniart, Local tame lifting for GL(n) II: wildly ramified supercuspidals, Astérisque 254 Soc. Math. France, 1999.

[7] C. Bushnell and G. Henniart, The local Langlands conjecture for $G L(2)$, Grundlerhen der math. Wissenschaften 335 Springer, 2006.

[8] V. Ennola, On the characters of the finite unitary groups, Ann. Acad. Scien. Fenn., 323 (1963),1-35.

[9] Y. Flicker, Stable and labile base change for $U(2)$, Duke Math. J., 49 (1982), 691729 .

[10] Y. Flicker, On distinguished representations, J.Reine Angew. Math. 418 (1991), 134-172.

[11] A. Fröhlich, Principal orders and embedding of local fields in algebras, Proc. London Math. Soc., 54 (1987), 247-266.

[12] J. HAKim And F. Murnaghan, Two types of distinguished supercuspidal representations, Int. Math. Res. Not., 35 (2002), 1857-1889.

[13] H. Jacquet and R. Langlands, Automorphic forms on $G L(2)$, Lectures Notes in Math. 114, Springer Verlag, 1970.

[14] R. Koтtwitz, Rational conjugacy classes on reductive groups, Duke Math. J., 49 (1982), 785-806.

[15] P.C. Kutzko and P.J. SAlly JR., All supercuspidal representations of $S L_{\ell}$ over a $p$-adic field are induced, Proceedings of the Utah conference on representation Theory, Progress in Math., 40 (1983), 185-196.

[16] J.-P. Labesse And R. LAnglands, $L$-indistinguishability for $S L(2)$, Can. J. Math., 31 (1979), 726-785.

[17] L. Morris, Tamely ramified supercuspidal representations, Ann. scient. Ec. Norm. Sup., 29 (1996), 639-667.

[18] J. Rogawski, Automorphic representations of unitary groups in three variables, Ann. of Math. Studies 123 Princeton University Press, 1990.

[19] T.A. Springer, Characters of special groups, Seminar on Algebraic groups and related finite groups 1968/69, Lectures Notes in Math., 131 (1970), 121-166.

[20] S. Stevens, Double coset decompositions and intertwining, Manuscripta Math., 106 (2001), 349-364.

Département de Mathématiques et U.M.R. 8628 DU C.N.R.S., Université ParisSud, BÂtiment 425, 91405 Orsay Cedex, France

E-mail address: Laure.Blasco@math.u-psud.fr 